\title{
Short-range neutrinoless double beta decay mechanisms
}

\author{
Lukas Graf, ${ }^{1,2,3, *}$ Frank F. Deppisch, ${ }^{1, \dagger}$ Francesco Iachello, ${ }^{2, \hbar}$ and Jenni Kotila, \\ ${ }^{1}$ Department of Physics and Astronomy, University College London, \\ Gower Street, London WC1E 6BT, United Kingdom \\ ${ }^{2}$ Center for Theoretical Physics, Sloane Physics Laboratory, Yale University, \\ New Haven, Connecticut 06520-8120, USA \\ ${ }^{3}$ Perimeter Institute for Theoretical Physics, Caroline Street North, Waterloo, Ontario N2L 2Y5, Canada \\ ${ }^{4}$ Finnish Institute for Educational Research, University of Jyväskylä, P.O. Box 35, Jyväskylä FI-40014, Finland
}

(Received 29 September 2018; published 26 November 2018)

\begin{abstract}
Neutrinoless double beta decay can significantly help to shed light on the issue of nonzero neutrino mass, as the observation of this lepton-number-violating process would imply that neutrinos are Majorana particles. However, the underlying interaction does not have to be as simple as the standard neutrino mass mechanism. The entire variety of neutrinoless double beta decay mechanisms can be approached effectively. In this work, we focus on a theoretical description of short-range effective contributions to neutrinoless double beta decay, which are equivalent to nine-dimensional effective operators incorporating the appropriate field content. We give a detailed derivation of the nuclear matrix elements and phase-space factors corresponding to individual terms of the effective Lagrangian. Using these, we provide general formulas for the neutrinoless double beta decay half-life and angular correlation of the outgoing electrons.
\end{abstract}

DOI: $10.1103 /$ PhysRevD.98.095023

\section{INTRODUCTION}

While the Standard Model (SM) gauge group $S U(3)_{C} \times$ $S U(2)_{L} \times U(1)_{Y}$ perfectly explains the interactions we observe, its breaking also provides masses to the charged fermions via the Higgs mechanism. The discovery of the Higgs at the Large Hadron Collider (LHC) [1,2] allows us to probe and test this mass mechanism in the SM. Yet, neutrinos continue to evade our understanding, as only lefthanded neutrinos exist in the SM and they therefore cannot acquire a so-called Dirac mass like the other SM fermions. Neutrino oscillation experiments [3] have unambiguously shown, though, that at least two of the three known neutrino species have finite masses and, while they are not sensitive to the absolute neutrino masses, they point to mass scales of the order of $10^{-2}$ to $5 \times 10^{-2} \mathrm{eV}$. In addition, cosmological observations set an upper limit on the sum of neutrino masses $\Sigma m_{\nu} \lesssim 0.15 \mathrm{eV}$ [4], assuming the standard cosmological model and with the exact value depending on the observational data considered.

\footnotetext{
*lukas.graf.14@ucl.ac.uk

f.deppisch@ucl.ac.uk

francesco.iachello@yale.edu

§jenni.kotila@jyu.fi
}

Published by the American Physical Society under the terms of the Creative Commons Attribution 4.0 International license. Further distribution of this work must maintain attribution to the author(s) and the published article's title, journal citation, and DOI. Funded by SCOAP ${ }^{3}$.
Neutrinos could be of the Dirac type, as the other SM fermions are, but this requires a new right-handed neutrino $\nu_{R}$ and tiny Yukawa couplings $\lesssim 10^{-12}$, which is rather unnatural. Because the right-handed neutrinos would be completely sterile with respect to the SM gauge interactions, it is, on the other hand, theoretically indicated that they acquire a Majorana mass $M$ of the form $M \bar{\nu}_{L} C \bar{\nu}_{L}^{T}$. It is generically expected to be of the order of a large new physics scale $\Lambda_{\mathrm{NP}} \approx M$ associated with the breaking of lepton number $L$ symmetry. Via the Yukawa couplings between left- and right-handed neutrinos, it will induce an effective dimension-5 operator, $\Lambda_{\mathrm{NP}}^{-1}(L L H H)$ [5], where $L$ and $H$ represent the $S U(2)_{L}$ left-handed lepton and the Higgs doublets, respectively. After electroweak (EW) symmetry breaking, a small effective Majorana mass $m_{\nu} \sim$ $m_{\mathrm{EW}}^{2} / \Lambda_{\mathrm{NP}}$ is generated for the active neutrinos. This corresponds to the famous seesaw mechanism [6-10], with a scale $\Lambda_{\mathrm{NP}}$ naturally of the order of $10^{14} \mathrm{GeV}$ to explain the light neutrino masses $m_{\nu} \approx 0.1 \mathrm{eV}$.

While the most prominent scenario, the high-scale seesaw mechanism, is not the only possibility to generate light neutrino masses, there are numerous other ways by incorporating lepton number violation (LNV) at low scales in secluded sectors, at higher loop order, and when allowing higher-dimensional effective interactions beyond the Weinberg operator. If the $L$ breaking occurs closer to the EW scale, higher-dimensional $L$-breaking operators will be important for phenomenology, and, specifically, they will potentially induce neutrinoless double beta $0 \nu \beta \beta$ decay. 
The search for $0 \nu \beta \beta$ decay is the most sensitive approach to probe Majorana neutrino masses. The experimentally most stringent lower limit on the decay half-life $T_{1 / 2}$ is derived using the xenon isotope ${ }_{54}^{136} \mathrm{Xe}$,

$$
T_{1 / 2}^{\mathrm{Xe}} \equiv T_{1 / 2}\left({ }_{54}^{136} \mathrm{Xe} \rightarrow{ }_{56}^{136} \mathrm{Ba}+e^{-} e^{-}\right) \gtrsim 10^{26} \mathrm{yr} .
$$

However, Majorana neutrino masses are not the only element of beyond-the-SM physics which can induce it. As hinted at above, other mechanisms of $0 \nu \beta \beta$ decay where the LNV originates from LNV masses and couplings of new particles appearing in various possible extensions of the SM. The same couplings and states will also induce light neutrino masses due to the Schechter-Valle black box argument [11], but the resulting contribution will not be necessarily dominant. Instead, we consider the $0 \nu \beta \beta$ decay rate by expressing high-scale new physics contributions in terms of effective low-energy operators [12-15]. As a basis of our subsequent discussion, we provide a brief overview of the possible effective contact interactions at the Fermi scale $m_{F} \approx 100 \mathrm{MeV}$ at which $0 \nu \beta \beta$ decay occurs. These are likewise triggered by effective SM invariant operators violating $\Delta L=2$ of dimension $5,7,9,11$, etc. Figure 1 schematically shows the contribution of such operators. They can, in general, be categorized in two main classes:

(i) Long-range transitions proceeding through the exchange of a light neutrino.-This includes the so-called standard neutrino mass mechanism via Majorana neutrinos in Fig. 1(a), for which the decay rate can be estimated by dimensional analysis as $\Gamma_{m_{\nu}}^{0 \nu \beta \beta} \sim m_{\nu}^{2} G_{F}^{4} m_{F}^{2} Q_{\beta \beta}^{5} \sim\left(m_{\nu} / 0.1 \mathrm{eV}\right)^{2}\left(10^{26} \mathrm{yr}\right)^{-1}$.

Here, $G_{F}$ is the Fermi coupling constant of the SM charged current interaction, and the phase space available to the two electrons scales as $Q_{\beta \beta}^{5}$ with $Q_{\beta \beta}=\mathcal{O}(1 \mathrm{MeV})$ for typical double beta decay nuclear transitions. Alternatively, in models with exotic interactions incorporating right-chiral neutrinos, no mass insertion is required; cf. Fig. 1(b). In the SM with only left-handed neutrinos, these operators violate $\Delta L=2$, and they incorporate a helicity flip through the inclusion of a Higgs field. In this case, the decay rate is estimated as $\Gamma_{\mathrm{LR}}^{0 \nu \beta \beta} \sim v^{2} \Lambda_{O_{7}}^{-6} G_{F}^{2} m_{F}^{4} Q_{\beta \beta}^{5} \sim$ $\left(10^{5} \mathrm{GeV} / \Lambda_{O_{7}}\right)^{6}\left(10^{26} \mathrm{yr}\right)^{-1}$, with the SM Higgs vacuum expectation value $v$ and the scale $\Lambda_{O_{7}}$ of the dim-7 operator.

(ii) Short-range transitions with no mediating particle lighter than $\approx 100 \mathrm{MeV}$.-As contact interactions with six external fermions, they are of dimension 9 and higher odd dimensions. For a dim-9 operator, the decay rate can be estimated as $\Gamma_{\mathrm{SR}}^{0 \nu \beta \beta} \sim$ $\Lambda_{O_{9}}^{-10} m_{F}^{6} Q_{\beta \beta}^{5} \sim\left(5 \mathrm{TeV} / \Lambda_{O_{9}}\right)^{10}\left(10^{26} \mathrm{yr}\right)^{-1}$, with the associated operator scale $\Lambda_{O_{9}}$. Thus, $0 \nu \beta \beta$ decay probes LNV physics around the TeV scale. The most prominent scenario where such an operator is generated is through the inclusion of heavy sterile neutrinos [16]. In the above classification, we do not include the case where additional light states are either mediating the decay or are emitted in it (e.g., Majorons).

Probing exotic $\Delta L=2$ transitions is crucial for our understanding of how light neutrinos acquire their tiny masses. As indicated above, if, for example, exotic shortrange contributions were to be observed in upcoming experiments, it would indicate that the origin of light neutrino masses is around the $\mathrm{TeV}$ scale. Likewise, the nonobservation of $0 \nu \beta \beta$ puts strong constraints on neutrino mass mechanisms close to the EW scale. It is not just neutrino physics that can be probed, though. Operators violating $\Delta L=2$, or the underlying physics responsible for them, can also erase an asymmetry between the number of leptons and antileptons throughout the thermal history of the early Universe. Together with the sphaleron transitions in the SM violating the sum of total baryon and lepton numbers $(B+L)$, this will also erase an asymmetry between baryons and antibaryons. The rate of this washout can be related to the half-life of $0 \nu \beta \beta$ decay for a given operator. The observation of nonstandard $0 \nu \beta \beta$ decay mechanisms can thus generally falsify baryogenesis mechanisms operating at scales above the EW scale [17,18]. A similar argument applies to other process probing lepton number violation around the $\mathrm{TeV}$ scale, such as searches for same-sign dileptons at the LHC $[19,20]$.

Before discussing the exotic short-range contributions of our interest, we remind the reader that the mass mechanism of $0 \nu \beta \beta$ decay is sensitive to the effective Majorana neutrino mass

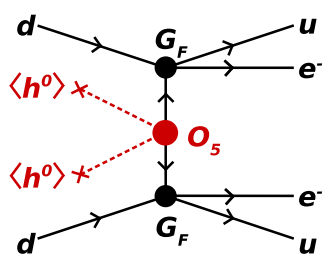

(a)

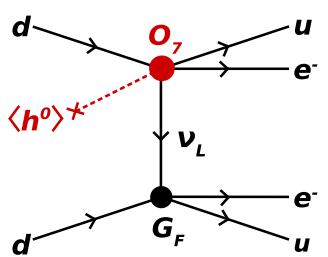

(b)

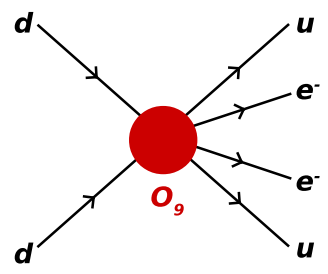

(c)

FIG. 1. Contributions to $0 \nu \beta \beta$ decay from effective higher-dimensional LNV operators: (a) 5-dim Weinberg operator (standard mass mechanism), (b) 7-dim operator leading to long-range contribution, and (c) 9-dim operator leading to short-range contribution. Adapted from Ref. [17]. 


$$
m_{\nu}=\sum_{i=1}^{3} U_{e i}^{2} m_{\nu_{i}} \equiv m_{e e},
$$

where the sum is over all light neutrinos with masses $m_{\nu_{i}}$, weighted by the square of the charged-current leptonic mixing matrix $U$. This quantity is equal to the $(e e)$ entry of the Majorana neutrino mass matrix. The inverse $0 \nu \beta \beta$ decay half-life in a given isotope can then be expressed by

$$
T_{1 / 2}^{-1}=\left|\frac{m_{\nu}}{m_{e}}\right|^{2} G_{\nu}\left|\mathcal{M}_{\nu}\right|^{2},
$$

where $G_{\nu}$ is the phase-space factor (PSF) and $\mathcal{M}_{\nu}$ the corresponding nuclear matrix element (NME) of the process. The normalization with respect to the electron mass $m_{e}$ yields a small dimensionless parameter $\epsilon_{\nu}=$ $m_{\nu} / m_{e}$. The current experimental results lead to a limit $m_{\nu} \lesssim 0.06-0.17 \mathrm{eV}$ [21], with an uncertainty due to the different NMEs in various nuclear structure models. Future experiments will probe $m_{\nu} \approx 0.02 \mathrm{eV}$, corresponding to the lowest value for an inverse hierarchy of the light neutrino states. A popular modification is through the inclusion of light sterile neutrinos with masses in the range from $\mathrm{eV}$ to $\mathrm{MeV}$ [22-25], in which case the half-life is still given by Eq. (3) but with different masses $m_{\nu_{i}}$ and couplings $U_{e i}$ [26-29].

The NMEs of the nuclear $0 \nu \beta \beta$ transitions are notoriously difficult to calculate, and limits derived from $0 \nu \beta \beta$ decay are affected for any contribution. Detailed treatments using different nuclear structure model approaches can, for example, be found in Refs. [30-39]. Despite tremendous efforts to improve the nuclear theory calculation, the latest matrix elements obtained using various approaches differ in many cases by factors of $\sim(2-3)$. Experimentally, the most stringent bounds on $0 \nu \beta \beta$ decay are currently from ${ }^{76} \mathrm{Ge}$ [40] and ${ }^{136} \mathrm{Xe}$ [21]. The results presented below are using the recent results in ${ }^{76} \mathrm{Ge}$ of $T_{1 / 2}^{\mathrm{Ge}} \geq 5.3 \times 10^{25} \mathrm{yr}$ and in ${ }^{136} \mathrm{Xe}$ of $T_{1 / 2}^{\mathrm{Xe}} \geq 1.07 \times 10^{26} \mathrm{yr}$ at $90 \%$ confidence level (C.L.). Planned future experiments searching for $0 \nu \beta \beta$ decay are expected to reach sensitivities of the order of $T_{1 / 2} \approx 10^{27} \mathrm{yr}$. For example, the recent comparative analysis [41] quotes a discovery sensitivity at $3 \sigma$ of $T_{1 / 2}^{\mathrm{Xe}}=$ $4.1 \times 10^{27} \mathrm{yr}$ for the planned nEXO experiment [42]. For more details on the effective $0 \nu \beta \beta$ interaction, see, for example, the review [43] and references therein. General up-to-date reviews of $0 \nu \beta \beta$ decay and associated physics can be found in Ref. [44], while a more specific recent review on $0 \nu \beta \beta$ NMEs is available in Ref. [45].

Besides the light and heavy neutrino exchange, exotic long-range mechanisms have received the most attention so far [46-50]. This is reasonable, as the underlying SM invariant operators already occur at dimension 7, and, as mentioned above, $0 \nu \beta \beta$ decay is sensitive to high scales of the order of $\Lambda_{O_{7}} \approx 10^{5} \mathrm{GeV}$. It is important to note, though, that, due to the intrinsic helicity-flip involved, such operators are typically strongly constrained by the smallness of the light neutrino masses. For example, in the popular left-right symmetric model [51-54], the effective dim-7 operator is constrained by the small Yukawa coupling $y_{\nu}$ of the active neutrino to the heavy sterile neutrino $N, y_{\nu} v=\sqrt{m_{\nu} M_{N}}$, due to neutrino mass generation via the seesaw mechanism. If the right-handed neutrinos acquire masses of the order of the left-right symmetry-breaking scale $\Lambda_{\mathrm{LR}}$, this effectively changes the operator contribution $v / \Lambda_{O_{7}}^{3} \rightarrow m_{\nu}^{1 / 2} / \Lambda_{\mathrm{LR}}^{5 / 2}$. The $0 \nu \beta \beta$ decay rate then scales as $\Gamma_{\mathrm{LR}}^{0 \nu \beta \beta} \sim\left(m_{\nu} / 0.1 \mathrm{eV}\right)\left(5 \mathrm{TeV} / \Lambda_{\mathrm{LR}}\right)^{5}\left(10^{26} \mathrm{yr}\right)^{-1}$, and the long-range mechanism in fact probes the $\mathrm{TeV}$ scale as well. While not all scenarios feature such a strong tree-level suppression, it has been shown that it is not straightforward to have a dim-7 operator for which the exotic long-range contribution dominates over the standard mass mechanism [55]. In our work, we instead focus on short-range mechanisms that often do not suffer such a strong suppression at a similar level. In the left-right symmetry example, the short-range contribution originates from two right-handed charged currents with $\mathcal{O}(1)$ gauge strength interactions. In both long- and short-range contributions, the half-life triggered by a single mechanism may be generically expressed similarly to Eq. (3):

$$
T_{1 / 2}^{-1}=\left|\epsilon_{I}\right|^{2} G_{I}\left|\mathcal{M}_{I}\right|^{2},
$$

where $G_{I}$ is the nuclear PSF and $\mathcal{M}_{I}$ the NME, both generally depending on the Lorentz structure of the effective operator in question. The coupling constant $\epsilon_{I}$ parametrizes the underlying particle physics dynamics, e.g., the couplings to and the masses of the heavy states integrated out. NMEs and PSFs for dimension- 6 operators were given in Ref. [50]. In this paper, we present a detailed derivation of NMEs and PSFs for dimension-9 effective operators, and we try to clarify and improve various aspects of the previous treatment [13]. In our calculation, we include additional NMEs that become important when the latest values of the nucleon form factors are taken into account. Moreover, we provide more realistic PSFs calculated using the exact radial wave functions, and we present the single electron energy and angular correlation distributions for these exotic $0 \nu \beta \beta$ decay mechanisms.

The paper is arranged as follows. After presenting the general effective Lagrangian at the quark level in Sec. II, we outline the calculation of the $0 \nu \beta \beta$ differential decay rate in Sec. III. We then give the derivation of the NMEs in Sec. IV. Section V details the calculation of the leptonic PSFs. The results of these calculations are then combined in Sec. VI to give explicit expressions for the decay rate and angular correlations. Limits on the effective couplings $\epsilon_{I}$ are also derived therein, assuming one contribution is 
different from zero at a time. Section VII contains some concluding remarks.

\section{EFFECTIVE PARTICLE PHYSICS LAGRANGIAN}

The contributions to $0 \nu \beta \beta$ decay can be parametrized by effective operators of dimension 6 and 9 [12,13], corresponding to short-range and long-range interactions, respectively. The general Lagrangian of $0 \nu \beta \beta$ decay consists of long-range and short-range parts, corresponding to pointlike vertices at the Fermi scale $\approx 100 \mathrm{MeV}$.

In this work, we concentrate on the short-range contributions for which the general effective interaction Lagrangian schematically reads [13]

$$
\mathcal{L}_{\mathrm{SR}}=\frac{G_{F}^{2}}{2 m_{p}} \sum_{\text {chiralities }}\left[\epsilon_{1}^{\cdot} J_{\circ} J_{\circ} j_{\circ}+\epsilon_{2}^{\cdot} J_{\circ}^{\mu \nu} J_{\circ \mu \nu} j_{\circ}+\epsilon_{3}^{\cdot} J_{\circ}^{\mu} J_{\circ \mu} j_{\circ}+\epsilon_{4}^{\cdot} J_{\circ}^{\mu} J_{\circ \mu \nu} j^{\nu}+\epsilon_{5}^{\cdot} J_{\circ}^{\mu} J_{\circ} j_{\mu}\right],
$$

where the sum and the place holders $\circ$ indicate that the currents involved can have different chiralities and there is a separate effective coupling $\epsilon_{i}^{*}$ for each such combination. Specifically, the hadronic and leptonic currents in Eq. (5) are

$$
\begin{gathered}
J_{R / L}=\bar{u}\left(1 \pm \gamma_{5}\right) d, \quad J_{R / L}^{\mu}=\bar{u} \gamma^{\mu}\left(1 \pm \gamma_{5}\right) d, \\
J_{R / L}^{\mu \nu}=\bar{u} \sigma_{\mu \nu}\left(1 \pm \gamma_{5}\right) d, \\
j_{R / L}=\bar{e}\left(1 \pm \gamma_{5}\right) e^{c}, \quad j^{\mu}=\bar{e} \gamma^{\mu} \gamma_{5} e^{c},
\end{gathered}
$$

with $\sigma_{\mu \nu}=\frac{i}{2}\left[\gamma_{\mu}, \gamma_{\nu}\right]$. The fields $u, d$, and $e$ are fourcomponent Dirac spinor operators representing the up quark, down quark, and electron, respectively. The field $e^{c}=\mathrm{Ce}$ denotes the charge conjugate, corresponding to the fact that all lepton currents violate the electron lepton number by two units. While the currents involved in Eq. (5) can have different chiralities as denoted in Eq. (6), the results will not depend on many of the specific choices.

As is convention, the Lagrangian Eq. (5) is normalized by the factor $G_{F}^{2} /\left(2 m_{p}\right)$ with the Fermi constant $G_{F}$ and the proton mass $m_{p}$. As a result, the effective coupling constants $\epsilon_{i}$ are dimensionless.

In the Lagrangian Eq. (5) one does not have to consider all possible combinations of the chiralities of the currents, as some of them are redundant or vanish. In order to prevent any confusion about the basis of low-energy dimension-9 operators we are considering, we spell these explicitly out in Table I. Each operator is labeled in the same way as the corresponding effective coupling in Eq. (5), i.e., $\mathcal{O}_{i}^{*} \sim \epsilon_{i}^{*}$, where the superscript specifies the chiralities of the particular bilinears in their respective order. We explicitly identify the equivalent (and thus redundant) operators, and we omit operators $\mathcal{O}_{2}^{R L L}, \mathcal{O}_{2}^{L R L}, \mathcal{O}_{2}^{R L R}$, and $\mathcal{O}_{2}^{L R R}$, which trivially vanish, because of the identity

$$
\begin{aligned}
& {\left[\bar{u} \sigma^{\mu \nu}\left(1+\gamma_{5}\right) d\right]\left[\bar{u} \sigma_{\mu \nu}\left(1-\gamma_{5}\right) d\right]} \\
& \quad \equiv\left[\bar{u} \sigma^{\mu \nu}\left(1-\gamma_{5}\right) d\right]\left[\bar{u} \sigma_{\mu \nu}\left(1+\gamma_{5}\right) d=0 .\right.
\end{aligned}
$$

Similarly, the Lagrangian Eq. (5) does not contain any terms with vector, tensor, or axial-tensor electron currents, as $\bar{e} \gamma^{\mu} e^{c}=0$ and $\bar{e} \sigma_{\mu \nu}\left(1 \pm \gamma_{5}\right) e^{c}=0$, due to the Pauli exclusion principle.

The 24 operators in Table I are linearly independent and form a complete basis of nine-dimensional operators invariant under the gauge group $S U(3)_{C} \otimes U(1)_{Q}$ and contributing to $0 \nu \beta \beta$ decay. We also show explicitly the assumed contractions of the color indices $i$ and $j$, although these are trivial, as always the quarks within the same Lorentz bilinear are contracted. The total number of

\begin{tabular}{|c|c|}
\hline $\mathcal{O}_{1}^{R R R}$ & {$\left[\bar{u}^{i}\left(1+\gamma_{5}\right) d_{i}\right]\left[\bar{u}^{j}\left(1+\gamma_{5}\right) d_{j}\right]\left[\bar{e}\left(1+\gamma_{5}\right) e^{c}\right]$} \\
\hline $\mathcal{O}_{1}^{R R L}$ & {$\left[\bar{u}^{i}\left(1+\gamma_{5}\right) d_{i}\right]\left[\bar{u}^{j}\left(1+\gamma_{5}\right) d_{j}\right]\left[\bar{e}\left(1-\gamma_{5}\right) e^{c}\right]$} \\
\hline $\mathcal{O}_{1}^{L R R} \equiv \mathcal{O}_{1}^{R L R}$ & {$\left[\bar{u}^{i}\left(1-\gamma_{5}\right) d_{i}\right]\left[\bar{u}^{j}\left(1+\gamma_{5}\right) d_{j}\right]\left[\bar{e}\left(1+\gamma_{5}\right) e^{c}\right]$} \\
\hline $\mathcal{O}_{1}^{L R L} \equiv \mathcal{O}_{1}^{R L L}$ & {$\left[\bar{u}^{i}\left(1-\gamma_{5}\right) d_{i}\right]\left[\bar{u}^{j}\left(1+\gamma_{5}\right) d_{j}\right]\left[\bar{e}\left(1-\gamma_{5}\right) e^{c}\right]$} \\
\hline $\mathcal{O}_{1}^{L L R}$ & {$\left[\bar{u}^{i}\left(1-\gamma_{5}\right) d_{i}\right]\left[\bar{u}^{j}\left(1-\gamma_{5}\right) d_{j}\right]\left[\bar{e}\left(1+\gamma_{5}\right) e^{c}\right]$} \\
\hline $\mathcal{O}_{1}^{L L L}$ & {$\left[\bar{u}^{i}\left(1-\gamma_{5}\right) d_{i}\right]\left[\bar{u}^{j}\left(1-\gamma_{5}\right) d_{j}\right]\left[\bar{e}\left(1-\gamma_{5}\right) e^{c}\right]$} \\
\hline $\mathcal{O}_{2}^{R R R}$ & {$\left[\bar{u}^{i} \sigma^{\mu \nu}\left(1+\gamma_{5}\right) d_{i}\right]\left[\bar{u}^{j} \sigma_{\mu \nu}\left(1+\gamma_{5}\right) d_{j}\right]\left[\bar{e}\left(1+\gamma_{5}\right) e^{c}\right]$} \\
\hline $\mathcal{O}_{2}^{R R L}$ & {$\left[\bar{u}^{i} \sigma^{\mu \nu}\left(1+\gamma_{5}\right) d_{i}\right]\left[\bar{u}^{j} \sigma_{\mu \nu}\left(1+\gamma_{5}\right) d_{j}\right]\left[\bar{e}\left(1-\gamma_{5}\right) e^{c}\right]$} \\
\hline $\mathcal{O}_{2}^{L L R}$ & {$\left[\bar{u}^{i} \sigma^{\mu \nu}\left(1-\gamma_{5}\right) d_{i}\right]\left[\bar{u}^{j} \sigma_{\mu \nu}\left(1-\gamma_{5}\right) d_{j}\right]\left[\bar{e}\left(1+\gamma_{5}\right) e^{c}\right]$} \\
\hline $\mathcal{O}_{2}^{L L L}$ & {$\left[\bar{u}^{i} \sigma^{\mu \nu}\left(1-\gamma_{5}\right) d_{i}\right]\left[\bar{u}^{j} \sigma_{\mu \nu}\left(1-\gamma_{5}\right) d_{j}\right]\left[\bar{e}\left(1-\gamma_{5}\right) e^{c}\right]$} \\
\hline $\mathcal{O}_{3}^{R R R}$ & {$\left[\bar{u}^{i} \gamma^{\mu}\left(1+\gamma_{5}\right) d_{i}\right]\left[\bar{u}^{j} \gamma_{\mu}\left(1+\gamma_{5}\right) d_{j}\right]\left[\bar{e}\left(1+\gamma_{5}\right) e^{c}\right]$} \\
\hline $\mathcal{O}_{3}^{R R L}$ & {$\left[\bar{u}^{i} \gamma^{\mu}\left(1+\gamma_{5}\right) d_{i}\right]\left[\bar{u}^{j} \gamma_{\mu}\left(1+\gamma_{5}\right) d_{j}\right]\left[\bar{e}\left(1-\gamma_{5}\right) e^{c}\right]$} \\
\hline $\mathcal{O}_{3}^{L R R} \equiv \mathcal{O}_{3}^{R L R}$ & {$\left[\bar{u}^{i} \gamma^{\mu}\left(1-\gamma_{5}\right) d_{i}\right]\left[\bar{u}^{j} \gamma_{\mu}\left(1+\gamma_{5}\right) d_{j}\right]\left[\bar{e}\left(1+\gamma_{5}\right) e^{c}\right]$} \\
\hline $\mathcal{O}_{3}^{L R L} \equiv \mathcal{O}_{3}^{R L L}$ & {$\left[\bar{u}^{i} \gamma^{\mu}\left(1-\gamma_{5}\right) d_{i}\right]\left[\bar{u}^{j} \gamma_{\mu}\left(1+\gamma_{5}\right) d_{j}\right]\left[\bar{e}\left(1-\gamma_{5}\right) e^{c}\right]$} \\
\hline $\mathcal{O}_{3}^{L L R}$ & {$\left[\bar{u}^{i} \gamma^{\mu}\left(1-\gamma_{5}\right) d_{i}\right]\left[\bar{u}^{j} \gamma_{\mu}\left(1-\gamma_{5}\right) d_{j}\right]\left[\bar{e}\left(1+\gamma_{5}\right) e^{c}\right]$} \\
\hline $\mathcal{O}_{3}^{L L L}$ & {$\left[\bar{u}^{i} \gamma^{\mu}\left(1-\gamma_{5}\right) d_{i}\right]\left[\bar{u}^{j} \gamma_{\mu}\left(1-\gamma_{5}\right) d_{j}\right]\left[\bar{e}\left(1-\gamma_{5}\right) e^{c}\right]$} \\
\hline $\mathcal{O}_{4}^{R R}$ & {$\left[\bar{u}^{i} \gamma^{\mu}\left(1+\gamma_{5}\right) d_{i}\right]\left[\bar{u}^{j} \sigma_{\mu \nu}\left(1+\gamma_{5}\right) d_{j}\right]\left[\bar{e} \gamma^{\nu} \gamma_{5} e^{c}\right]$} \\
\hline $\mathcal{O}_{4}^{R L}$ & {$\left[\bar{u}^{i} \gamma^{\mu}\left(1+\gamma_{5}\right) d_{i}\right]\left[\bar{u}^{j} \sigma_{\mu \nu}\left(1-\gamma_{5}\right) d_{j}\right]\left[\bar{e} \gamma^{\nu} \gamma_{5} e^{c}\right]$} \\
\hline $\mathcal{O}_{4}^{L R}$ & {$\left[\bar{u}^{i} \gamma^{\mu}\left(1-\gamma_{5}\right) d_{i}\right]\left[\bar{u}^{j} \sigma_{\mu \nu}\left(1+\gamma_{5}\right) d_{j}\right]\left[\bar{e} \gamma^{\nu} \gamma_{5} e^{c}\right]$} \\
\hline $\mathcal{O}_{4}^{L L}$ & {$\left[\bar{u}^{i} \gamma^{\mu}\left(1-\gamma_{5}\right) d_{i}\right]\left[\bar{u}^{j} \sigma_{\mu \nu}\left(1-\gamma_{5}\right) d_{j}\right]\left[\bar{e} \gamma^{\nu} \gamma_{5} e^{c}\right]$} \\
\hline $\mathcal{O}_{5}^{R R}$ & {$\left[\bar{u}^{i} \gamma^{\mu}\left(1+\gamma_{5}\right) d_{i}\right]\left[\bar{u}^{j}\left(1+\gamma_{5}\right) d_{j}\right]\left[\bar{e} \gamma_{\mu} \gamma_{5} e^{c}\right]$} \\
\hline $\mathcal{O}_{5}^{R L}$ & {$\left[\bar{u}^{i} \gamma^{\mu}\left(1+\gamma_{5}\right) d_{i}\right]\left[\bar{u}^{j}\left(1-\gamma_{5}\right) d_{j}\right]\left[\bar{e} \gamma_{\mu} \gamma_{5} e^{c}\right]$} \\
\hline $\mathcal{O}_{5}^{L R}$ & {$\left[\bar{u}^{i} \gamma^{\mu}\left(1-\gamma_{5}\right) d_{i}\right]\left[\bar{u}^{j}\left(1+\gamma_{5}\right) d_{j}\right]\left[\bar{e} \gamma_{\mu} \gamma_{5} e^{c}\right]$} \\
\hline $\mathcal{O}_{5}^{L L}$ & {$\left[\bar{u}^{i} \gamma^{\mu}\left(1-\gamma_{5}\right) d_{i}\right]\left[\bar{u}^{j}\left(1-\gamma_{5}\right) d_{j}\right]\left[\bar{e} \gamma_{\mu} \gamma_{5} e^{c}\right]$} \\
\hline
\end{tabular}
these operators agrees with the result we obtained as a consistency check from a calculation using the Hilbert

TABLE I. Basis of low-scale, nine-dimensional operators invariant under the $S U(3)_{C} \otimes U(1)_{Q}$ gauge group contributing to $0 \nu \beta \beta$ decay. 
series method [56,57] and with the results in Ref. [58]. Despite comments in the latter reference, we do not see the need to include operators containing quark bilinears transforming as color octets. These can be shown to be related by Fierz transformation to the herein presented operators with tensor Lorentz structure. For example, for operator $\mathcal{O}_{2}^{L L L}$, one can find the following Fierz identity:

$$
\begin{aligned}
\mathcal{O}_{2}^{L L L}= & {\left[\bar{u}^{i} \sigma^{\mu \nu}\left(1-\gamma_{5}\right) d_{i}\right]\left[\bar{u}^{j} \sigma_{\mu \nu}\left(1-\gamma_{5}\right) d_{j}\right] j_{L} } \\
= & 2\left[\bar{u}^{i}\left(1-\gamma_{5}\right) d_{j}\right]\left[\bar{u}^{j}\left(1-\gamma_{5}\right) d_{i}\right] j_{L} \\
& -\left[\bar{u}^{i}\left(1-\gamma_{5}\right) d_{i}\right]\left[\bar{u}^{j}\left(1-\gamma_{5}\right) d_{j}\right] j_{L} .
\end{aligned}
$$

If we further apply the $S U(3)_{C}$ color Fierz identity based on the well-known group-theoretical formula for the GellMann matrices $\lambda^{a}(a=1, \ldots, 8)$,

$$
\delta_{i j} \delta_{k l}=\frac{1}{3} \delta_{i l} \delta_{k j}+\frac{1}{2}\left(\lambda^{a}\right)_{i l}\left(\lambda_{a}\right)_{k j},
$$

on the first term on the right-hand side of Eq. (9), we get

$$
\begin{aligned}
\mathcal{O}_{2}^{L L L}= & {\left[\bar{u}^{i}\left(1-\gamma_{5}\right)\left(\lambda^{a}\right)_{i k} d^{k}\right]\left[\bar{u}^{j}\left(1-\gamma_{5}\right)\left(\lambda_{a}\right)_{j l} d^{l}\right] j_{L} } \\
& -\frac{1}{3}\left[\bar{u}^{i}\left(1-\gamma_{5}\right) d_{i}\right]\left[\bar{u}^{j}\left(1-\gamma_{5}\right) d_{j}\right] j_{L} \\
\equiv & {\left[\bar{u}^{i}\left(1-\gamma_{5}\right)\left(\lambda^{a}\right)_{i k} d^{k}\right]\left[\bar{u}^{j}\left(1-\gamma_{5}\right)\left(\lambda_{a}\right)_{j l} d^{l}\right] j_{L} } \\
& -\frac{1}{3} \mathcal{O}_{1}^{L L L} .
\end{aligned}
$$

Hence, we see that if the operator containing color octets (the first term on the right-hand side of the above equation) is neglected, for the fact that it does not contribute to $0 \nu \beta \beta$ decay as argued in Ref. [59], then the operator $\mathcal{O}_{2}^{L L}$ is equivalent to $-\frac{1}{3} \mathcal{O}_{1}^{L L L}$ and, thus, redundant. In a similar way, all the operators with tensor quark bilinears can be traded for operators containing color octets. If these are neglected, then only the operators consisting of (both leftand right-handed) scalar and vector color-singlet quark bilinears are left. We will nevertheless report on the direct limits on the tensor operators $\mathcal{O}_{2}^{*}$ for a comparison with previous literature and to be independent of the assumption that color nonsinglet currents do not contribute to $0 \nu \beta \beta$ decay.

\section{NEUTRINOLESS DOUBLE BETA DECAY RATE}

The differential rate of $0 \nu \beta \beta$ decay can be written as

$$
d \Gamma=2 \pi \overline{|\mathcal{R}|^{2}} \delta\left(E_{1}+E_{2}+E_{F}-E_{I}\right) \frac{d^{3} \mathbf{p}_{1}}{(2 \pi)^{3}} \frac{d^{3} \mathbf{p}_{2}}{(2 \pi)^{3}},
$$

where $\overline{|\mathcal{R}|^{2}}$ is the full matrix element of the $0 \nu \beta \beta$ decay process summed over the spin projections $s_{1}$ and $s_{2}$ of the electrons and the final nuclear state $S_{F}$. The 4-momenta of the outgoing electrons are $\left(E_{1}, \mathbf{p}_{1}\right),\left(E_{2}, \mathbf{p}_{2}\right)$, and $E_{F}$ and $E_{I}$ are the energies of the final and initial nuclei, respectively. The $Q_{\beta \beta}$ value of the transition, i.e., the kinetic energy release of the electrons, is given by $Q_{\beta \beta}=E_{I}-E_{F}-2 m_{e}$, with the electron mass $m_{e}=0.511 \mathrm{MeV}$. Here, we neglect the recoil energy of the final nucleus which is of the order of $Q_{\beta \beta}^{2} /\left(2 M_{A}\right)=\mathcal{O}(0.1 \mathrm{keV})$ for isotope masses $M_{A}$ of interest. Because of the overall rotational invariance and energy conservation, the differential rate can be expressed in terms of the energy $m_{e}<E_{1}<Q_{\beta \beta}+m_{e}$ of one of the electrons and the angle $0 \leq \theta \leq \pi$ between the two electrons, with $\cos \theta=\hat{\mathbf{p}}_{1} \cdot \hat{\mathbf{p}}_{2}$. ${ }^{1}$ The energy of the other electron is then determined as $E_{2}=Q_{\beta \beta}+2 m_{e}-E_{1}$, and the magnitudes of the electron 3-momenta are $p_{i} \equiv\left|\mathbf{p}_{i}\right|=\sqrt{E_{i}^{2}-m_{e}^{2}}$.

The full matrix element of the process can be formally expressed as

$$
\mathcal{R}=\left\langle\mathcal{O}_{F}^{+} e_{\mathbf{p}_{1} s_{1}} e_{\mathbf{p}_{2} s_{2}}\left|\mathcal{L}_{\mathrm{SR}}\right| \mathcal{O}_{I}^{+}\right\rangle
$$

Here, $\left\langle\mathcal{O}_{F}^{+} e_{\mathbf{p}_{1} s_{1}} e_{\mathbf{p}_{2} s_{2}}\right|$ denotes the final state composed of the $0^{+}$daughter nuclear state and the two emitted electrons and $\left|\mathcal{O}_{I}^{+}\right\rangle$the initial nuclear state. It is understood that the wave function of the two electrons in Eq. (13) is antisymmetrized; the same holds for the wave functions of the $\mathcal{O}_{I}^{+}$and $\mathcal{O}_{F}^{+}$states in terms of their constituent nucleons. In Eq. (13), we allow the most general form of the quark level Lagrangian Eq. (5) for $\mathcal{L}_{\mathrm{SR}}$, which we symbolically express as

$$
\mathcal{L}_{\mathrm{SR}}=\frac{G_{F}^{2}}{2 m_{p}} \sum_{K, \Xi} \varepsilon_{K} j_{K}^{\Xi} J_{K}^{\Xi} J_{K}^{\prime \Xi},
$$

where the summation over $K$ and $\Xi$ collectively denotes the different electron-quark-quark current combinations $j J J^{\prime}$ (including different chiralities) and the Lorentz contractions, respectively.

The evaluation of the matrix element $\mathcal{R}$ is rather complicated, since, in general, the leptonic part is nested with the hadronic part. For the long-range case, a detailed calculation was given by Doi et al. [46,47] and Tomoda [60]. Since the hadronic part is the product of two currents, a sum over a set of intermediate states $|\mathcal{N}\rangle$ must be performed. This is a daunting task, since for $0 \nu \beta \beta$ decay all states up to an energy $E \approx 100 \mathrm{MeV}$ contribute. It is therefore customary to treat the above summation in the closure approximation, i.e., sum over a complete set of states:

$$
\sum_{\mathcal{N}}\left\langle\mathcal{O}_{F}^{+}\left|J_{K}^{\Xi}\right| \mathcal{N}\right\rangle\left\langle\mathcal{N}\left|J_{K}^{\prime \Xi}\right| \mathcal{O}_{I}^{+}\right\rangle \approx\left\langle\mathcal{O}_{F}^{+}\left|J_{K}^{\Xi} J_{K}^{\prime \Xi}\right| \mathcal{O}_{I}^{+}\right\rangle
$$

\footnotetext{
${ }^{1}$ Throughout, we denote normalized vectors by $\hat{\mathbf{v}} \equiv \mathbf{v} /|\mathbf{v}|$.
} 
This approximation is very well justified in the case of short-range operators, as the intermediate transition occurs at very high energies $|\mathbf{q}| \approx 100 \mathrm{MeV}$, corresponding to the internucleon distance, compared to the nuclear transition itself at $Q_{\beta \beta} \approx 1 \mathrm{MeV}$.

Another problem is that the leptonic and hadronic parts are entangled. In order to disentangle them, an approximation is made wherein the electron wave functions are evaluated at the surface of the nucleus [46,61]. This approximation can be improved by using simplified nucleon wave functions and calculating the weighted average electron position [61], but the approximation we employ here does not introduce a sizable error.

Following this approach, the overall matrix element $\mathcal{R}$ is factorized into (i) the product of the leptonic matrix element that will be integrated over the two-electron phase space yielding the so-called PSF that depends only on the leptonic current and the electron wave function at the surface of the nucleus ${ }^{2}$ and (ii) the NME.

For the latter, we first reduce each nucleon current $J_{K}^{\Xi}$ to its nonrelativistic form by means of a Foldy-Wouthuysen (FW) transformation, then take the product of the two currents, and evaluate the matrix elements of the corresponding two-body operator in the nuclear many-body wave functions. In the FW transformation, we take terms to the order of $|\mathbf{q}| / m_{p}$. This is a good approximation, since the momentum transfer in the process is of the order of $|\mathbf{q}| \sim 100 \mathrm{MeV}$, and therefore $|\mathbf{q}| / m_{p} \sim 0.1$. In certain cases of enhanced form factors, higher-order terms in the products of hadronic currents are also taken into account.

Altogether, this yields the full matrix element

$$
\mathcal{R}=\frac{G_{F}^{2}}{2 m_{p}} \sum_{K, \Xi} \epsilon_{K}\left\langle e_{\mathbf{p}_{1} s_{1}}\left|j_{K}^{\Xi}\right| e_{\mathbf{p}_{2} s_{2}}^{c}\right\rangle\left\langle\mathcal{O}_{F}^{+}\left|J_{K}^{\Xi} J_{K}^{\Xi \Xi}\right| \mathcal{O}_{I}^{+}\right\rangle
$$

The leptonic matrix elements will be evaluated using the appropriate electron wave functions in Sec. V. The nucleon matrix elements are evaluated as discussed above, including appropriate $\mathbf{q}^{2}$-dependent form factors, in Secs. IVA and IV B and the nuclear matrix elements in Sec. IV C.

Putting together the PSFs and NMEs, one can write the fully differential rate for $0^{+} \rightarrow 0^{+} 0 \nu \beta \beta$ decay as [46-48]

$$
\frac{d^{2} \Gamma}{d E_{1} d \cos \theta}=C w\left(E_{1}\right)\left(a\left(E_{1}\right)+b\left(E_{1}\right) \cos \theta\right),
$$

with

$$
C=\frac{G_{F}^{4} m_{e}^{2}}{16 \pi^{5}}
$$

\footnotetext{
${ }^{2}$ In the associated $2 \nu \beta \beta$ decay, it will also depend on the outgoing neutrino wave functions.

${ }^{3}$ We note in passing that, for $0^{+} \rightarrow 2^{+} 0 \nu \beta \beta$ decay, there is an additional term in Eq. (17) of the form $c\left(E_{1}\right)\left(\cos ^{2} \theta-1 / 3\right)$.
}

$$
w\left(E_{1}\right)=E_{1} E_{2} p_{1} p_{2},
$$

where $E_{2}, p_{1}$, and $p_{2}$ are expressed as functions of $E_{1}$.

Following the notation of Refs. [46-48], the coefficient $1 / m_{p}$ appearing in Eq. (16) is included in the calculation of the nuclear matrix elements [see the following Eq. (55)], and a mass $m_{e}$ is added in the numerator to cancel the mass $m_{e}$ in the denominator of the so-called neutrino potential in Eq. (55).

The total decay rate $\Gamma$ and the decay half-life $T_{1 / 2}$ are then given by

$$
\Gamma=\frac{\ln 2}{T_{1 / 2}}=2 C \int_{m_{e}}^{Q_{\beta \beta}+m_{e}} d E_{1} w\left(E_{1}\right) a\left(E_{1}\right) .
$$

From Eq. (17), one can calculate the single electron energy distribution

$$
\frac{d \Gamma}{d E_{1}}=2 C w\left(E_{1}\right) a\left(E_{1}\right)
$$

and the energy-dependent angular correlation

$$
\alpha\left(E_{1}\right)=\frac{b\left(E_{1}\right)}{a\left(E_{1}\right)} .
$$

Introducing the integrated quantities

$$
\begin{aligned}
A & =\int_{m_{e}}^{Q_{\beta \beta}+m_{e}} d E_{1} w\left(E_{1}\right) a\left(E_{1}\right), \\
B & =\int_{m_{e}}^{Q_{\beta \beta}+m_{e}} d E_{1} w\left(E_{1}\right) b\left(E_{1}\right)
\end{aligned}
$$

and their ratio $K=B / A$, one obtains the angular distribution

$$
\frac{d \Gamma}{d \cos \theta}=\frac{\Gamma}{2}(1+K \cos \theta) .
$$

Although both the single electron energy distribution and the angular correlation require dedicated experimental setups, we calculate them nonetheless, since they contain important information on the underlying mechanism.

\section{NUCLEAR MATRIX ELEMENTS}

\section{A. From quarks to nucleons}

We are interested in matrix elements induced by the quark bilinears appearing in the Lagrangian Eq. (5), i.e., by the left- and right-handed scalar, vector, and tensor quark currents. Considering the nucleon isodoublet $N=\left(\begin{array}{l}p \\ n\end{array}\right)$, the nucleon matrix elements of these color singlet quark currents have according to [62] the following structure: 


$$
\begin{array}{r}
\left\langle p\left|\bar{u}\left(1 \pm \gamma_{5}\right) d\right| n\right\rangle=\bar{N} \tau^{+}\left[F_{S}\left(q^{2}\right) \pm F_{P S}\left(q^{2}\right) \gamma_{5}\right] N^{\prime} \\
\left\langle p\left|\bar{u} \gamma^{\mu}\left(1 \pm \gamma_{5}\right) d\right| n\right\rangle \\
=\bar{N} \tau^{+}\left[F_{V}\left(q^{2}\right) \gamma^{\mu}-i \frac{F_{W}\left(q^{2}\right)}{2 m_{p}} \sigma^{\mu \nu} q_{\nu}\right] N^{\prime} \\
\pm \bar{N} \tau^{+}\left[F_{A}\left(q^{2}\right) \gamma^{\mu} \gamma_{5}-\frac{F_{P}\left(q^{2}\right)}{2 m_{p}} \gamma_{5} q^{\mu}\right] N^{\prime} \\
\left\langle p\left|\bar{u} \sigma^{\mu \nu}\left(1 \pm \gamma_{5}\right) d\right| n\right\rangle=\bar{N} \tau^{+}\left[J^{\mu \nu} \pm \frac{i}{2} \epsilon^{\mu \nu \rho \sigma} J_{\rho \sigma}\right] N^{\prime}
\end{array}
$$

Here we define

$$
\begin{aligned}
J^{\mu \nu}= & F_{T_{1}}\left(q^{2}\right) \sigma^{\mu \nu}+i \frac{F_{T_{2}}\left(q^{2}\right)}{m_{p}}\left(\gamma^{\mu} q^{\nu}-\gamma^{\nu} q^{\mu}\right) \\
& +\frac{F_{T_{3}}\left(q^{2}\right)}{m_{p}^{2}}\left(\sigma^{\mu \rho} q_{\rho} q^{\nu}-\sigma^{\nu \rho} q_{\rho} q^{\mu}\right),
\end{aligned}
$$

and $\tau^{+}$is the isospin-raising operator, transforming a neutron into a proton. The matrix elements are, in general, functions of the neutron and proton momenta $p_{n}=p_{N^{\prime}}$ and $p_{p}=p_{N}$, respectively, and the momentum transfer entering the form factors is defined as $q=p_{p}-p_{n}$. In Eq. (26), we omit the induced scalar and axial-tensor terms; the corresponding currents can be safely neglected, because they vanish in the isospin-symmetric limit [63]. Moreover, they are suppressed by a factor of $1 / m_{p}$, and they are not enhanced by a pion resonance.

Important ingredients in Eqs. (25)-(27) are the $q^{2}$ dependent form factors $F_{X}\left(q^{2}\right)$ with $X \in\{S, P S, V, W, A$, $\left.P, T_{1}, T_{2}, T_{3}\right\}$. We parametrize these except $F_{P S}\left(q^{2}\right)$ and $F_{P}\left(q^{2}\right)$ in the so-called dipole form,

$$
F_{X}\left(q^{2}\right)=\frac{g_{X}}{\left(1+q^{2} / m_{X}^{2}\right)^{2}}
$$

where the coupling constants $g_{X}$ give the value of the form factor at zero momentum transfer, $g_{X}=F_{X}(0)$.

For example, the vector form factor can be experimentally determined from the electromagnetic form factor and from the conserved vector current hypothesis:

$$
\begin{aligned}
& F_{V}\left(q^{2}\right)=\frac{g_{V}}{\left(1+q^{2} / m_{V}^{2}\right)^{2}}, \\
& g_{V}=1, \quad m_{V}=0.84 \mathrm{GeV} .
\end{aligned}
$$

This parametrization provides a good description of $F_{V}\left(q^{2}\right)$ in the range $0 \leq|q| \leq 200 \mathrm{MeV}$ of interest in $0 \nu \beta \beta$ decay. A better parametrization, important for large $q^{2} \gtrsim 1 \mathrm{GeV}^{2}$, is given in Ref. [64], but it is of no interest for the purposes of the present paper.

The induced form factor $F_{W}\left(q^{2}\right)$ can also be determined from the experiment, since it is related to the Pauli form factor $F_{2}\left(q^{2}\right)$ [64] and to the isovector anomalous magnetic moment of the nucleon:

$$
\begin{aligned}
& F_{W}\left(q^{2}\right)=\frac{g_{W}}{\left(1+q^{2} / m_{W}^{2}\right)^{2}}, \\
& g_{W}=\mu_{p}-\mu_{n}=3.70, \quad m_{W}=m_{V}=0.84 \mathrm{GeV},
\end{aligned}
$$

where $\mu_{p}-\mu_{n}$ is the anomalous isovector magnetic moment of the proton and neutron.

The axial-vector form factor can also be parametrized in dipole form, and it is obtained from the experiment:

$$
\begin{aligned}
& F_{A}\left(q^{2}\right)=\frac{g_{A}}{\left(1+q^{2} / m_{A}^{2}\right)^{2}}, \\
& g_{A}=1.269, \quad m_{A}=1.09 \mathrm{GeV} .
\end{aligned}
$$

The value of $g_{A}$ is determined in neutron decay [65], and $m_{A}$ is obtained from neutrino scattering [66].

The induced form factor $F_{P}\left(q^{2}\right)$ cannot be directly obtained from the experiment. We use the parametrization suggested in Ref. [16], based on the partially conserved axial-vector current hypothesis:

$$
F_{P}\left(q^{2}\right)=\frac{g_{A}}{\left(1+q^{2} / m_{A}^{2}\right)^{2}} \frac{1}{1+q^{2} / m_{\pi}^{2}} \frac{4 m_{p}^{2}}{m_{\pi}^{2}}\left(1-\frac{m_{\pi}^{2}}{m_{A}^{2}}\right),
$$

with the pion mass $m_{\pi}=0.138 \mathrm{GeV}$.

From Eq. (33), we have $g_{P} \equiv F_{P}(0)=231$. This formula is consistent with a recent analysis in the chiral perturbation theory [67], which gives $g_{P}=233$, and with recent measurements in muon capture, which give $F_{P}\left(q^{2}\right) \frac{\left|\mathbf{q}^{2}\right|}{2 m_{p}}$ at $|\mathbf{q}|=0.88 m_{\mu}$, where $m_{\mu}=0.105 \mathrm{GeV}$ is the muon mass. The calculated value is 8.0 , while the measured value is $8.06 \pm 0.55$ [68].

A considerable amount of attention has been devoted recently to the form factors $F_{S}\left(q^{2}\right)$ and $F_{P S}\left(q^{2}\right)$, in particular, to the values at zero momentum transfer, $g_{S}=$ $F_{S}(0)$ and $g_{P S}=F_{P S}(0)$. Quoted values are $g_{S}=1.02 \pm$ 0.11 and $g_{P S}=349 \pm 9$ [69]. Not much is known about the $q^{2}$ dependence; for the scalar form factor, which, in the Breit frame, is the Fourier transform of the matter distribution, a reasonable parametrization is in the dipole form with $g_{S}=1$ and $m_{S}=m_{V}=0.84 \mathrm{GeV}$ :

$$
\begin{aligned}
& F_{S}\left(q^{2}\right)=\frac{g_{S}}{\left(1+q^{2} / m_{S}^{2}\right)^{2}} \\
& g_{S}=1, \quad m_{S}=m_{V}=0.84 \mathrm{GeV} .
\end{aligned}
$$

The value of the pseudoscalar form factor $F_{P S}\left(q^{2}\right)$ diverges at $q^{2}=0$ in the chiral limit, and the results of lattice calculations depend on the extrapolation procedure. We take 


$$
\begin{aligned}
& F_{P S}\left(q^{2}\right)=\frac{g_{P S}}{\left(1+q^{2} / m_{P S}^{2}\right)^{2}} \frac{1}{1+q^{2} / m_{\pi}^{2}}, \\
& g_{P S}=349, \quad m_{P S}=m_{V}=0.84 \mathrm{GeV}
\end{aligned}
$$

The question of whether or not the value of $g_{P S}$ is enhanced as in Ref. [69] is beyond the scope of this paper. The parametrization (35) reduces to the simple monopole form $1 /\left(1+q^{2} / m_{\pi}^{2}\right)$ used in the chiral perturbation theory, but it includes the finite size of the nucleon.

No experimental information is available for the tensor form factors. Reference [69] quotes a value of $0.987 \pm 0.055$ for $F_{T_{1}}(0) \equiv g_{T_{1}}$. An old calculation [62] estimates, from the MIT bag model, $F_{T_{1}}(0) \equiv g_{T_{1}}=1.38, F_{T_{2}}(0) \equiv g_{T_{2}}=-3.30$, and $F_{T_{3}}(0) \equiv g_{T_{3}}=1.34$. In this paper, we take

$$
\begin{aligned}
& F_{T_{i}}\left(q^{2}\right)=\frac{g_{T_{i}}}{\left(1+q^{2} / m_{T_{i}}^{2}\right)^{2}}, \\
& m_{T_{i}}=m_{V}=0.84 \mathrm{GeV},
\end{aligned}
$$

with $g_{T_{1}}=1$ (and $g_{T_{2}}=-3.30$ and $g_{T_{3}}=1.34$ estimated from Ref. [62]). The two form factors $F_{T_{2}}\left(q^{2}\right)$ and $F_{T_{3}}\left(q^{2}\right)$ do not enter the results of this paper but are quoted here for completeness.

\section{B. Nonrelativistic expansion}

To obtain the nuclear matrix elements of interest, we have to calculate the nonrelativistic expansion of the above nucleon matrix elements. This form is obtained by a FoldyWouthuysen transformation [70,71], which is an expansion in powers of the velocity $v / c$ or, equivalently, in $|\mathbf{p}| / m_{p}$.

The resulting expressions are summarized in the following section, where we use the spatial momentum difference $\mathbf{q}=$ $\mathbf{p}_{p}-\mathbf{p}_{n}$ and momentum sum $\mathbf{Q}=\mathbf{p}_{p}+\mathbf{p}_{n}$. Particular terms will be listed according to the order in $|\mathbf{q}| / m_{p}$, where we perform the expansion up to and including terms of the order of $|\mathbf{q}| / m_{p}$, except for terms incorporating $F_{P}\left(q^{2}\right)$ and $F_{P S}\left(q^{2}\right)$, which are enhanced as discussed above. In these cases, we retain terms of the order of $\mathbf{q}^{2} / m_{p}^{2}$ and even higher.

Scalar bilinears. - The nonrelativistic expansion of the scalar and pseudoscalar nucleon current corresponding to $J_{S \pm P}=\left(1 \pm \gamma_{5}\right)$ can be written as

$$
J_{S \pm P}=F_{S}\left(q^{2}\right) I \pm \frac{F_{P S}\left(q^{2}\right)}{2 m_{p}} \boldsymbol{\sigma} \cdot \mathbf{q}+\cdots .
$$

Here, $I$ denotes the $2 \times 2$ identity matrix, and $\boldsymbol{\sigma}=$ $\left(\sigma_{1}, \sigma_{2}, \sigma_{2}\right)^{T}$ is the vector of Pauli matrices, both operating in the spin space of the nucleon.

Vector bilinears. - The vector currents corresponding to $J_{V \pm A}^{\mu}=\gamma^{\mu}\left(1 \pm \gamma_{5}\right)$ have four different components (vector, axial-vector, and induced pseudoscalar and weak magnetism), which can be nonrelativistically expanded as follows:

$$
\begin{aligned}
J_{V \pm A}^{\mu}= & g^{\mu i}\left[\mp F_{A}\left(q^{2}\right) \sigma_{i}-\frac{F_{V}\left(q^{2}\right)}{2 m_{p}} \mathbf{Q}_{i} I+\frac{F_{V}\left(q^{2}\right)+F_{W}\left(q^{2}\right)}{2 m_{p}} i(\boldsymbol{\sigma} \times \mathbf{q})_{i} \pm \frac{F_{P}\left(q^{2}\right)}{4 m_{p}^{2}} q_{i} \boldsymbol{\sigma} \cdot \mathbf{q}\right] \\
& +g^{\mu 0}\left[F_{V}\left(q^{2}\right) I \pm \frac{F_{A}\left(q^{2}\right)}{2 m_{p}} \boldsymbol{\sigma} \cdot \mathbf{Q} \mp \frac{F_{P}\left(q^{2}\right)}{4 m_{p}^{2}} q_{0} \boldsymbol{\sigma} \cdot \mathbf{q}\right]+\cdots
\end{aligned}
$$

Tensor bilinears. - The nonzero nuclear components corresponding to the tensor bilinears $J_{T \pm T_{5}}^{\mu \nu}=\sigma^{\mu \nu}\left(1 \pm \gamma_{5}\right)$ are

$$
J_{T \pm T_{5}}^{\mu \nu}=F_{T_{1}}\left(q^{2}\right) g^{\mu j} g^{\nu k} \varepsilon_{i j k} \sigma^{i}+\left(g^{\mu i} g^{\nu 0}-g^{\mu 0} g^{\nu i}\right) T_{i} \pm \frac{i}{2} \varepsilon^{\mu \nu \rho \sigma}\left[\left(g_{\rho i} g_{\sigma 0}-g_{\rho 0} g_{\sigma i}\right) T^{i}+F_{T_{1}}\left(q^{2}\right) g_{\rho m} g_{\sigma n} \varepsilon^{m n i} \sigma_{i}\right]+\cdots
$$

where

$$
\mathcal{J}_{\bar{K}}^{\Xi}(\mathbf{x})=\sum_{a} \tau_{+}^{a} \delta\left(\mathbf{x}-\mathbf{r}_{a}\right) J_{K, a}^{\Xi},
$$

$T^{i}=\frac{i}{2 m_{p}}\left[\left(F_{T_{1}}\left(q^{2}\right)-2 F_{T_{2}}\left(q^{2}\right)\right) q^{i} I+F_{T_{1}}\left(q^{2}\right)\left(\boldsymbol{\sigma}_{a} \times \mathbf{Q}\right)^{i}\right]$

Terms containing the momentum sum $\mathbf{Q}$ are called recoil terms [48].

The nuclear currents can be obtained from Eqs. (37)(40) by summing over all neutrons, located at positions $\mathbf{r}_{a}$, in the initial nucleus as where $J_{K, a}^{\Xi}$ denotes any of the nucleon currents. ${ }^{4}$

The short-range $0 \nu \beta \beta$ decay transition involves two such currents, each transforming one neutron into a proton. Having the five different terms in the effective Lagrangian Eq. (5), we thus need to evaluate five different products of nucleon currents in the nonrelativistic expansion. Furthermore, we need to sum over all neutrons in the

\footnotetext{
${ }^{4}$ For positron emission, $\tau_{+}$is replaced by $\tau_{-}$, and the sum is over protons.
} 
initial nucleus, and the corresponding nuclear transition operators can be expressed as

$$
\mathcal{H}_{K}(\mathbf{x}, \mathbf{y})=\sum_{a \neq b} \tau_{+}^{a} \tau_{+}^{b} \delta\left(\mathbf{x}-\mathbf{r}_{a}\right) \delta\left(\mathbf{y}-\mathbf{r}_{b}\right) \Pi_{K, a b}^{\Xi},
$$

with the products of the relevant nucleon currents $\Pi_{K, a b}^{\Xi}$ given in Appendix A. In the case of the terms 1, 2, and 3, the nuclear transition operator has no free Lorentz index, whereas for terms 4 and 5, there remains one free index that is contracted with that of the electron current.

\section{From nucleons to the nucleus}

The final and most challenging step in the determination of the $0 \nu \beta \beta$ NMEs concerns the calculation of the matrix elements at the nuclear level. This requires an understanding of nuclear structure, and, given the highly complex nature of the many-body problem, it is not solvable from first principles. Above, we have constructed the shortrange nuclear $0 \nu \beta \beta$ transition operators using the general nucleon operator with their $\mathbf{q}^{2}$ and, thus, distance dependence parametrized by experimentally constrained form functions. We now define the nuclear matrix elements as

$$
\mathcal{M}_{K} \equiv\left\langle\mathcal{O}_{F}^{+}\left|\mathcal{H}_{K}\right| \mathcal{O}_{I}^{+}\right\rangle
$$

with the transition operator given in Eq. (42) and where $\left\langle\mathcal{O}_{F}^{+}\right|$and $\left\langle\mathcal{O}_{I}^{+}\right|$denote the wave functions of the final and initial nuclear state under consideration, respectively. In principle, we would also need the wave functions of the intermediate states formed by a single beta-decay-like transition from one nucleon current. Exploiting the completeness of all intermediate states, we instead use the closure approximation in directly calculating the above matrix element. This approximation is very well justified in the case of short-range operators, as the intermediate transition occurs at very high energies $|q| \approx 100 \mathrm{MeV}$, corresponding to the internucleon distance, compared to the nuclear transition itself at $Q_{\beta \beta} \approx 1 \mathrm{MeV}$.

We concentrate on $0^{+} \rightarrow 0^{+}$transitions in this paper. In this case, all terms containing an odd number of $\boldsymbol{\sigma}$ and/or an odd number of $\mathbf{q}, \mathbf{Q}$ occurrences in $\Pi_{i}$ vanish, due to angular momentum and parity selection rules, when only $S_{1 / 2}-S_{1 / 2}$ wave approximation of the electron wave functions is assumed. Using the results in Appendix A, the matrix elements for the five short-range operators can be collected. In each case, we keep track of signs corresponding to different combinations of chiralities. For the first three operators (i.e., those proportional to $\epsilon_{1}, \epsilon_{2}$, and $\epsilon_{3}$ ), three sign possibilities are presented, and they correspond to the following combinations of chiralities (in this order): $R R, L L$, and $(1 / 2)(R L+L R)$. For the fourth and fifth operators (those proportional to $\epsilon_{4}$ and $\epsilon_{5}$ ), a row of four signs is shown, as in those cases the two hadronic currents have different Lorentz structures, and thus all four possible combinations of chiralities have to be considered (in this order): $R R, L L, R L$, and $L R$. To keep the expressions simple, when all three or four signs are the same, we show only a single sign. Using this notation, the matrix elements for the five different short-range operators read

$$
\begin{aligned}
& \mathcal{M}_{1}= g_{S}^{2} \mathcal{M}_{F}(++-) \frac{g_{P S}^{2}}{12 m_{p}^{2}}\left(\mathcal{M}_{G T}^{\prime P P}-\mathcal{M}_{T}^{\prime P P}\right), \\
& \mathcal{M}_{2}=-2 g_{T_{1}}^{2} \mathcal{M}_{G T}, \\
& \mathcal{M}_{3}=g_{V}^{2} \mathcal{M}_{F}(--+) g_{A}^{2} \mathcal{M}_{G T}^{A A}(++-) \\
& \times \frac{g_{A} g_{P}}{6 m_{p}^{2}}\left(\mathcal{M}_{G T}^{\prime A P}-\mathcal{M}_{T}^{\prime A P}\right) \\
&+\frac{\left(g_{V}+g_{W}\right)^{2}}{12 m_{p}^{2}}\left(\mathcal{M}_{G T}^{\prime}+\frac{1}{2} \mathcal{M}_{T}^{\prime}\right) \\
&(--+) \frac{g_{P}^{2}}{24 m_{p}^{4}}\left(\mathcal{M}_{G T}^{\prime \prime P P}-\mathcal{M}_{T}^{\prime \prime P P}\right),
\end{aligned}
$$

$$
\begin{aligned}
\mathcal{M}_{4}^{\mu}= & (--++) i g^{\mu 0} g_{A} g_{T_{1}} \mathcal{M}_{G T}^{A} \\
& (++--) i g^{\mu 0} \frac{g_{P} g_{T_{1}}}{12 m_{p}^{2}}\left(\mathcal{M}_{G T}^{\prime P}-\mathcal{M}_{T}^{\prime P}\right), \\
\mathcal{M}_{5}^{\mu}= & g^{\mu 0} g_{S} g_{V} \mathcal{M}_{F} \\
& (++--) g^{\mu 0} \frac{g_{A} g_{P S}}{12 m_{p}^{2}}\left(\tilde{\mathcal{M}}_{G T}^{A P}-\tilde{\mathcal{M}}_{T}^{A P}\right) \\
& (--++) g^{\mu 0} \frac{g_{P} g_{P S}}{24 m_{p}^{3}}\left(\mathcal{M}_{G T}^{q_{q} P P}-\mathcal{M}_{T}^{\prime q_{0} P P}\right) .
\end{aligned}
$$

In these expressions, we have kept all terms to the order of 1 . In $\mathcal{M}_{3}$, we have retained also the term proportional to $\left(F_{V}\left(q^{2}\right)+F_{W}\left(q^{2}\right)\right)^{2}$, which is a bit smaller but may still represent an important contribution of the respective operator. We have also separated out from the form factors $F_{X}\left(q^{2}\right)$ the so-called charges, i.e., the values at $q^{2}=0$ and $F_{X}(0) \equiv g_{X}$. The $q$ dependence is then given by (here $\left.X \in\left\{S, V, W, T_{1}, T_{2}, T_{3}\right\}\right)$

$$
\tilde{h}\left(q^{2}\right)=\frac{1}{\left(1+q^{2} / m_{V}^{2}\right)^{4}} .
$$

We treat separately the $A, P$, and $P S$ couplings which have a different $q$ dependence. If the axial-vector coupling $A$ is present in the first or second power, the $q$ dependence reads

$$
\tilde{h}_{A}\left(q^{2}\right)=\frac{1}{\left(1+q^{2} / m_{V}^{2}\right)^{2}} \frac{1}{\left(1+q^{2} / m_{A}^{2}\right)^{2}}
$$




$$
\tilde{h}_{A A}\left(q^{2}\right)=\frac{1}{\left(1+q^{2} / m_{A}^{2}\right)^{4}},
$$

respectively.

Similarly, if a single power of pseudoscalar coupling in combination with the axial-vector coupling $A$ or some other coupling $X$ is present, then we have

$$
\begin{aligned}
& \tilde{h}_{A P}\left(q^{2}\right)=\frac{1}{\left(1+q^{2} / m_{A}^{2}\right)^{4}} \frac{1}{\left(1+q^{2} / m_{\pi}^{2}\right)}, \\
& \tilde{h}_{P}\left(q^{2}\right)=\frac{1}{\left(1+q^{2} / m_{V}^{2}\right)^{4}} \frac{1}{\left(1+q^{2} / m_{\pi}^{2}\right)},
\end{aligned}
$$

respectively, while in the case of the second power of pseudoscalar coupling the $q$ dependence has the form

$$
\tilde{h}_{P P}\left(q^{2}\right)=\frac{1}{\left(1+q^{2} / m_{V}^{2}\right)^{4}} \frac{1}{\left(1+q^{2} / m_{\pi}^{2}\right)^{2}} .
$$

The Fermi $(F)$, Gamow-Teller $(G T)$, and tensor $(T)$ matrix elements appearing in Eqs. (44)-(48) can be calculated in any nuclear structure model $[31,33,72]$. We follow in this article the formulation of Refs. [16,31], where the two-body transition operator $H$ is constructed in momentum space as the product of the so-called neutrino potential $v(q)$ times the form factors $\tilde{h}\left(q^{2}\right)$. Since we consider short-range mechanisms with a $\delta$ function in configuration space, the Fourier transform is a constant, and the neutrino potential in momentum space is $[16,31]$

$$
v(q)=\frac{2}{\pi} \frac{1}{m_{e} m_{p}},
$$

where we have used the standard normalization. Incidentally, for the long-range mechanism, the neutrino potential is

$$
v(q)=\frac{2}{\pi} \frac{1}{q(q+\tilde{A})},
$$

where $\tilde{A}$ is the closure energy. This formulation allows one therefore to calculate simultaneously all matrix elements, short and long range, by simply specifying the neutrino potential.

As a further aside, to do calculations in coordinate space, one simply takes the Fourier-Bessel transforms of the product of the neutrino potential $v$ times the form factor $\tilde{h}$ :

$$
h(r)=\frac{2}{\pi} \int_{0}^{\infty} j_{\lambda}\left(q^{2}\right) \frac{1}{m_{e} m_{p}} \tilde{h}(q) q^{2} \mathrm{~d} q,
$$

where $\lambda=0$ for Fermi and Gamow-Teller contributions and $\lambda=2$ for a tensor contribution.

Finally, an additional improvement is the introduction of short-range correlations (SRCs) in the nuclear structure calculation. These are of crucial importance for short-range nonstandard mechanisms, and they can be taken into account by multiplying the potential $v(r)$ in coordinate space by a correlation function $f(r)$ squared. The most commonly used correlation function is the Jastrow function

$$
f_{J}(r)=1-c e^{-a r^{2}}\left(1-b r^{2}\right)
$$

with $a=1.1 \mathrm{fm}^{-2}, b=0.68 \mathrm{fm}^{-2}$, and $c=1$ for the phenomenological Miller-Spencer parametrization [73] and $a=1.59 \mathrm{fm}^{-2}, b=1.45 \mathrm{fm}^{-2}$, and $c=0.92$ for the Argonne parametrization [74]. Since the formulation described above is in momentum space, we take SRCs into account by using the Fourier-Bessel transform of $f_{J}(r)$.

Introducing

$$
h_{\circ}\left(q^{2}\right)=\frac{2}{\pi} \frac{1}{m_{e} m_{p}} \tilde{h}_{\circ}\left(q^{2}\right)
$$

(where the placeholder $\circ$ is used to note that the same redefinition is used for all the above defined types of $q$ dependencies) and the notation

$$
\left\langle H_{a b}\right\rangle=\left\langle\mathcal{O}_{F}^{+}\left|\sum_{a \neq b} \tau_{a}^{+} \tau_{b}^{+} H_{a b}\right| \mathcal{O}_{I}^{+}\right\rangle,
$$

where $H_{a b}$ denotes any two-body operator, we can write the Fermi $\left(\mathcal{M}_{F}\right)$ and Gamow-Teller $\left(\mathcal{M}_{G T}\right)$ matrix elements appearing in Eqs. (44)-(48) as

$$
\begin{gathered}
\mathcal{M}_{F}=\left\langle h\left(q^{2}\right)\right\rangle, \\
\mathcal{M}_{G T}=\left\langle h\left(q^{2}\right)\left(\boldsymbol{\sigma}_{a} \cdot \boldsymbol{\sigma}_{b}\right)\right\rangle .
\end{gathered}
$$

When the Gamow-Teller matrix element comes with one or two powers of the axial-vector coupling, we define

$$
\begin{gathered}
\mathcal{M}_{G T}^{A}=\left\langle h_{A}\left(q^{2}\right)\left(\boldsymbol{\sigma}_{a} \cdot \boldsymbol{\sigma}_{b}\right)\right\rangle, \\
\mathcal{M}_{G T}^{A A}=\left\langle h_{A A}\left(q^{2}\right)\left(\boldsymbol{\sigma}_{a} \cdot \boldsymbol{\sigma}_{b}\right)\right\rangle .
\end{gathered}
$$

In the third short-range operator, matrix elements $\mathcal{M}_{G T}^{\prime}$ and $\mathcal{M}_{T}^{\prime}$ appear, which are defined as

$$
\begin{gathered}
\frac{1}{m_{p}^{2}} \mathcal{M}_{G T}^{\prime}=\left\langle\frac{\mathbf{q}^{2}}{m_{p}^{2}} h\left(q^{2}\right)\left(\boldsymbol{\sigma}_{a} \cdot \boldsymbol{\sigma}_{b}\right)\right\rangle \\
\frac{1}{m_{p}^{2}} \mathcal{M}_{T}^{\prime}=\left\langle\frac{1}{m_{p}^{2}}\left[\mathbf{q}^{2}-\frac{1}{3}\left(\mathbf{q} \cdot \hat{\mathbf{r}}_{a b}\right)^{2}\right] h\left(q^{2}\right) \mathbf{S}_{a b}\right\rangle .
\end{gathered}
$$

Since $q \sim 100 \mathrm{MeV}$ in $0 \nu \beta \beta$ decay, these terms are suppressed by a factor of $\mathcal{O}(0.01)$ relative to the standard terms $\mathcal{M}_{G T}$ and $\mathcal{M}_{T}$. However, the enhancement of the corresponding form factor partly compensates this; therefore, 
we include them. These matrix elements can be easily calculated, since the neutrino potential is just a function of $\mathbf{q}^{2}$.

Similarly, the matrix elements $\mathcal{M}_{G T}^{\prime P}, \mathcal{M}_{T}^{\prime P}$ and $\mathcal{M}_{G T}^{\prime A P}$, $\mathcal{M}_{T}^{\prime A P}$ are given by

$$
\begin{gathered}
\frac{1}{m_{p}^{2}} \mathcal{M}_{G T}^{\prime P}=\left\langle\frac{\mathbf{q}^{2}}{m_{p}^{2}} h_{P}\left(q^{2}\right)\left(\boldsymbol{\sigma}_{a} \cdot \boldsymbol{\sigma}_{b}\right)\right\rangle, \\
\frac{1}{m_{p}^{2}} \mathcal{M}_{T}^{\prime P}=\left\langle\frac{1}{m_{p}^{2}}\left[\mathbf{q}^{2}-\frac{1}{3}\left(\mathbf{q} \cdot \hat{\mathbf{r}}_{a b}\right)^{2}\right] h_{P}\left(q^{2}\right) \mathbf{S}_{a b}\right\rangle
\end{gathered}
$$

and

$$
\begin{gathered}
\frac{1}{m_{p}^{2}} \mathcal{M}_{G T}^{\prime A P}=\left\langle\frac{\mathbf{q}^{2}}{m_{p}^{2}} h_{A P}\left(q^{2}\right)\left(\boldsymbol{\sigma}_{a} \cdot \boldsymbol{\sigma}_{b}\right)\right\rangle, \\
\frac{1}{m_{p}^{2}} \mathcal{M}_{T}^{\prime A P}=\left\langle\frac{1}{m_{p}^{2}}\left[\mathbf{q}^{2}-\frac{1}{3}\left(\mathbf{q} \cdot \hat{\mathbf{r}}_{a b}\right)^{2}\right] h_{A P}\left(q^{2}\right) \mathbf{S}_{a b}\right\rangle,
\end{gathered}
$$

respectively. These terms are also smaller by a factor of $\mathcal{O}(0.01)$ relative to the standard terms $\mathcal{M}_{G T}$ and $\mathcal{M}_{T}$. Nonetheless, this suppression is compensated by the enhancement of the $g_{P}$ form factor.

Next, we have terms $\mathcal{M}_{G T}^{\prime P P}$ and $\mathcal{M}_{T}^{\prime P P}$ contributing to the first short-range operator, which read

$$
\begin{gathered}
\frac{1}{m_{p}^{2}} \mathcal{M}_{G T}^{\prime P P}=\left\langle\frac{\mathbf{q}^{2}}{m_{p}^{2}} h_{P P}\left(q^{2}\right)\left(\boldsymbol{\sigma}_{a} \cdot \boldsymbol{\sigma}_{b}\right)\right\rangle \\
\frac{1}{m_{p}^{2}} \mathcal{M}_{T}^{\prime P P}=\left\langle\frac{1}{m_{p}^{2}}\left[\mathbf{q}^{2}-\frac{1}{3}\left(\mathbf{q} \cdot \hat{\mathbf{r}}_{a b}\right)^{2}\right] h_{P P}\left(q^{2}\right) \mathbf{S}_{a b}\right\rangle .
\end{gathered}
$$

These matrix elements are smaller by a factor of $\mathcal{O}(0.01)$. However, if the pseudoscalar coupling $g_{P S}$ is larger by 2 orders of magnitude as claimed in Ref. [69], these terms become comparable to those with the Fermi and GamowTeller matrix elements $\mathcal{M}_{F}$ and $\mathcal{M}_{G T}$ or even larger.

The matrix elements $\mathcal{M}_{G T}^{\prime \prime P P}$ and $\mathcal{M}_{T}^{\prime \prime P P}$ appearing in the third short-range operator can be written as

$$
\begin{gathered}
\frac{1}{m_{p}^{4}} \mathcal{M}_{G T}^{\prime \prime P P}=\left\langle\frac{\mathbf{q}^{4}}{m_{p}^{4}} h_{P P}\left(q^{2}\right)\left(\boldsymbol{\sigma}_{a} \cdot \boldsymbol{\sigma}_{b}\right)\right\rangle, \\
\frac{1}{m_{p}^{4}} \mathcal{M}_{T}^{\prime \prime P P}=\left\langle\frac{\mathbf{q}^{2}}{m_{p}^{4}}\left[\mathbf{q}^{2}-\frac{1}{3}\left(\mathbf{q} \cdot \hat{\mathbf{r}}_{a b}\right)^{2}\right] h_{P P}\left(q^{2}\right) \mathbf{S}_{a b}\right\rangle .
\end{gathered}
$$

Again, since $q \sim 100 \mathrm{MeV}$ in $0 \nu \beta \beta$ decay, these terms are smaller by a factor of $\mathcal{O}\left(10^{-4}\right)$ relative to the standard terms $\mathcal{M}_{G T}$ and $\mathcal{M}_{T}$. However, this suppression is again balanced by the enhancement of the form factor $g_{P}$, which appears here in the second power. These terms can be easily calculated, since the neutrino potential is just a function of $\mathbf{q}^{2}$.
The terms $\tilde{\mathcal{M}}_{G T}^{A P}$ and $\tilde{\mathcal{M}}_{T}^{A P}$, also called recoil terms, are defined as

$$
\begin{gathered}
\frac{1}{m_{p}^{2}} \tilde{\mathcal{M}}_{G T}^{A P}=\left\langle\frac{\mathbf{Q} \cdot \mathbf{q}}{m_{p}^{2}} h_{A P}\left(q^{2}\right)\left(\boldsymbol{\sigma}_{a} \cdot \boldsymbol{\sigma}_{b}\right)\right\rangle \\
\frac{1}{m_{p}^{2}} \tilde{\mathcal{M}}_{T}^{A P}=\left\langle\frac{1}{m_{p}^{2}}\left[\mathbf{Q} \cdot \mathbf{q}-\frac{1}{3}\left(\mathbf{Q} \cdot \hat{\mathbf{r}}_{a b}\right)\left(\mathbf{q} \cdot \hat{\mathbf{r}}_{a b}\right)\right] h_{A P}\left(q^{2}\right) \mathbf{S}_{a b}\right\rangle .
\end{gathered}
$$

Although these terms are suppressed by a factor of $\mathcal{O}(0.01)$, if the pseudoscalar coupling $g_{P S}$ is larger by 2 orders of magnitude, they will become important. These matrix elements are difficult to calculate, since the operator $\mathbf{Q}$ is not simply a function of $\mathbf{q}^{2}$. A good estimate can, however, be obtained by replacing $(\mathbf{Q} \cdot \mathbf{q}) / m_{p}^{2}$ with the expectation value in the state $\left|O_{I}^{+}\right\rangle,\left\langle\mathbf{Q} \cdot \mathbf{q} / m_{p}^{2}\right\rangle \sim 0.01$, in which case

$$
\frac{1}{m_{p}^{2}} \tilde{\mathcal{M}}_{G T}^{A P}=\left\langle\frac{\mathbf{Q} \cdot \mathbf{q}}{m_{p}^{2}}\right\rangle \mathcal{M}_{G T}^{A P}
$$

etc.

Finally, the terms $\mathcal{M}_{G T}^{\prime q_{0} P P}$ and $\mathcal{M}_{T}^{\prime q_{0} P P}$ appearing in the fifth matrix element read

$$
\begin{gathered}
\frac{1}{m_{p}^{3}} \mathcal{M}_{G T}^{\prime q_{0} P P}=\left\langle\frac{q_{0} \mathbf{q}^{2}}{m_{p}^{3}} h_{P P}\left(q^{2}\right)\left(\boldsymbol{\sigma}_{a} \cdot \boldsymbol{\sigma}_{b}\right)\right\rangle, \\
\frac{1}{m_{p}^{3}} \mathcal{M}_{T}^{\prime q_{0} P P}=\left\langle\frac{q_{0}}{m_{p}^{3}}\left[\mathbf{q}^{2}-\frac{1}{3}\left(\mathbf{q} \cdot \hat{\mathbf{r}}_{a b}\right)^{2}\right] h_{P P}\left(q^{2}\right) \mathbf{S}_{a b}\right\rangle .
\end{gathered}
$$

Since $q \sim 100 \mathrm{MeV}$ and $q_{0} \sim 10 \mathrm{MeV}$ in $0 \nu \beta \beta$ decay, these terms are smaller by a factor of $\mathcal{O}\left(10^{-4}\right)$ relative to the terms $\mathcal{M}_{G T}$ and $\mathcal{M}_{T}$. However, this suppression is balanced by the enhancement of the form factors $g_{P}$ and $g_{P S}$.

The above basic building blocks are written in terms of the Pauli spin operators $\boldsymbol{\sigma}$, the nucleon momenta difference $\mathbf{q}$ and sum $\mathbf{Q}$, and the direction unit vector between two nucleons, $\hat{\mathbf{r}}_{a b}=\mathbf{r}_{a b} /\left|\mathbf{r}_{a b}\right|$. Furthermore, we use $\mathbf{S}_{a b}=3\left(\boldsymbol{\sigma}_{a} \cdot \hat{\mathbf{r}}_{a b}\right)\left(\boldsymbol{\sigma}_{b} \cdot \hat{\mathbf{r}}_{a b}\right)-\left(\boldsymbol{\sigma}_{a} \cdot \boldsymbol{\sigma}_{b}\right)$.

Let us remark that, in the case of the short-range operator $\mathcal{O}_{1}$ incorporating scalar and pseudoscalar quark currents, the enhancement of the pseudoscalar form factor $g_{P S}$ can make the third-order term of the nonrelativistic expansion of the pseudoscalar current important. We anticipate this term to be of the order of $F_{P S}\left(q^{2}\right) \mathcal{O}\left(\mathbf{q}^{3} / m_{p}^{3}\right)$ and its product with the first-order pseudoscalar term of the expansion would give a contribution $F_{P S}^{2}\left(q^{2}\right) \mathcal{O}\left(\mathbf{q}^{4} / m_{p}^{4}\right) \sim$ $\mathcal{O}(1)$. This contribution is not listed in the above paragraphs, because only terms up to the order of $\mathbf{q} / m_{p}$ in the currents are considered in this paper. However, we 
conjecture that it will always be subdominant to the terms in Eqs. (71) and (72), which are larger by 2 orders of magnitude. The exact relative size of these contributions depends, of course, on the actual size of the corresponding NMEs, but there is no reason to believe that the NME involving the third-order term of the expansion should be exceptionally large.

\section{LEPTONIC PHASE-SPACE FACTORS}

The leptonic phase-space factors describe the atomic part of the physics involved in $0 \nu \beta \beta$ decay. They quantify the effect of the relativistic electrons emitted in the process. The position-dependent wave function of each electron can be expanded in terms of spherical waves,

$$
e_{\mathbf{p} s}(\mathbf{r})=e_{\mathbf{p} s}^{S_{1 / 2}}(\mathbf{r})+e_{\mathbf{p} s}^{P_{1 / 2}}(\mathbf{r})+\cdots
$$

where $\mathbf{p}$ is the asymptotic momentum of the electron at a long distance and $s$ denotes its spin projection. The $S_{1 / 2}$ and the $P_{1 / 2}$ waves on the right-hand side of the above expansion are, respectively, given by [48]

$$
\begin{aligned}
e_{\mathbf{p} s}^{S_{1 / 2}}(\mathbf{r}) & =\left(\begin{array}{c}
g_{-1}(E, r) \chi_{s} \\
f_{1}(E, r)(\boldsymbol{\sigma} \cdot \hat{\mathbf{p}}) \chi_{s}
\end{array}\right), \\
e_{\mathbf{p} s}^{P_{1 / 2}}(\mathbf{r}) & =i\left(\begin{array}{c}
g_{1}(E, r)(\boldsymbol{\sigma} \cdot \hat{\mathbf{r}})(\boldsymbol{\sigma} \cdot \hat{\mathbf{p}}) \chi_{s} \\
-f_{-1}(E, r)(\boldsymbol{\sigma} \cdot \hat{\mathbf{r}}) \chi_{s}
\end{array}\right),
\end{aligned}
$$

where $g_{\kappa}(E, r)$ and $f_{\kappa}(E, r)$ are the radial wave functions of the "large" and "small" components, respectively. The electron energy at asymptotically large distances is $E=\sqrt{\mathbf{p}^{2}+m_{e}^{2}}$, and its spin state is described by the two-dimensional spinor $\chi_{s}$. The Pauli matrices $\boldsymbol{\sigma}$ here operate in the electron spin space. The wave functions satisfy the asymptotic boundary condition

$$
\left(\begin{array}{c}
g_{\kappa}(E, r) \\
f_{\kappa}(E, r)
\end{array}\right) \stackrel{r \rightarrow \infty}{\longrightarrow} \frac{e^{-i \Delta_{\kappa}^{c}}}{p r}\left(\begin{array}{c}
\sqrt{\frac{E+m_{e}}{2 E}} \sin \left(p r+y \ln (2 p r)-\frac{1}{2} \pi l_{\kappa}+\Delta_{\kappa}^{c}\right) \\
\sqrt{\frac{E-m_{e}}{2 E}} \cos \left(p r+y \ln (2 p r)-\frac{1}{2} \pi l_{\kappa}+\Delta_{\kappa}^{c}\right)
\end{array}\right),
$$

where $\kappa= \pm\left(j+\frac{1}{2}\right), l_{\kappa}=j \pm \frac{1}{2}, y=\alpha Z_{F} E / p$, and $\Delta_{\kappa}^{c}$ is a phase shift. Here, $p=|\mathbf{p}|, \alpha$ is the fine structure constant, and $j$ is the total angular momentum of the electron. Inside the nucleus, the radial wave functions Eq. (81) can be expanded in $r$ approximated by the leading terms as

$$
\left(\begin{array}{c}
g_{-1}(E, r) \\
f_{1}(E, r)
\end{array}\right) \approx\left(\begin{array}{c}
A_{-1} \\
A_{+1}
\end{array}\right), \quad\left(\begin{array}{c}
g_{1}(E, r) \\
-f_{-1}(E, r)
\end{array}\right) \approx\left(\begin{array}{c}
A_{+1}\left[\frac{1}{2} \alpha Z_{F}+\frac{1}{3}\left(E+m_{e}\right) R_{A}\right] \frac{r}{R_{A}} \\
-A_{-1}\left[\frac{1}{2} \alpha Z_{F}+\frac{1}{3}\left(E-m_{e}\right) R_{A}\right] \frac{r}{R_{A}}
\end{array}\right)
$$

for $S_{1 / 2}$ and $P_{1 / 2}$ waves, respectively. Here $A_{\kappa}$ are normalization constants, and $R_{A}$ is the radius of the daughter nucleus. In the limit $Z_{F} \rightarrow 0$, the radial wave functions acquire the form of spherical Bessel functions, while the normalization constants become $A_{ \pm 1} \approx \sqrt{\left(E \mp m_{e}\right) /(2 E)}$. In our calculations, however, the above shown approximations are not employed, as we derive the phase-space factors using numerically calculated radial wave functions as described in Ref. [61]. Therein, a numerical solution is performed using a piecewise exact power series expansion of the radial wave functions. On top of the Coulomb potential of the daughter nucleus (with charge $Z_{F}$ ), $V(r)=-\alpha Z_{F} / r$, the nuclear size and electron cloud screening corrections are taken into account. As a result, the considered potential reads

$$
V(r)= \begin{cases}-\alpha Z_{F} \frac{3-\left(r / R_{A}\right)^{2}}{2 R_{A}} \times \varphi(r), & r<R \\ -\frac{\alpha Z_{F}}{r} \times \varphi(r), & r \geq R\end{cases}
$$

where $\varphi(r)$ is the Thomas-Fermi function taking into account the electron screening. The nontrivial $r$ dependence of the above potential for $r<R$ is a result of the finite nuclear size, when a uniform charge distribution in a sphere of radius $R_{A}=R_{0} A^{1 / 2}$ with $R_{0}=1.2 \mathrm{fm}$ is considered. In order to calculate the electron currents involved in $0 \nu \beta \beta$ decay, we in principle need to evaluate the wave functions at the position of the corresponding transition. To be exact, this would require the wave function of the nucleon undergoing the respective decay, ideally from the nuclear structure model, or using simplified harmonic oscillator wave functions [61].

Instead, we follow Ref. [61] and adopt the approximation of evaluating the electron wave function at the nuclear radius $r=R_{A}$ :

$$
\begin{gathered}
f_{ \pm 1}(E) \equiv f_{ \pm 1}\left(E, R_{A}\right), \\
g_{ \pm 1}(E) \equiv g_{ \pm 1}\left(E, R_{A}\right) .
\end{gathered}
$$


This choice reflects the fact that nucleons largely decay at the surface of the nucleus due to Pauli blocking of inner states.

For $0^{+} \rightarrow 0^{+}$transitions, parity-even nucleon operators need to be combined with $S_{1 / 2}-S_{1 / 2}$ and $P_{1 / 2}-P_{1 / 2}$ electron wave functions, while parity-odd operators need to be combined with $S_{1 / 2}-P_{1 / 2}$ wave functions. The calculation of the leptonic squared matrix elements are outlined in Appendix $\mathrm{B}$, and the results for $S_{1 / 2}-S_{1 / 2}$ wave functions are

$$
\begin{gathered}
\sum_{s_{1}, s_{2}}\left(\bar{e}_{1}\left(1+\gamma_{5}\right) e_{2}^{c}\right)\left(\bar{e}_{1}\left(1 \pm \gamma_{5}\right) e_{2}^{c}\right)^{\dagger} \frac{1-P_{e_{1} e_{2}}}{2} \\
=2\left[f_{11}^{(0)}+f_{11 \pm}^{(1)}\left(\hat{\mathbf{p}}_{1} \cdot \hat{\mathbf{p}}_{2}\right)\right], \\
\sum_{s_{1}, s_{2}}\left(\bar{e}_{1} \gamma_{\mu} \gamma_{5} e_{2}^{c}\right)\left(\bar{e}_{1} \gamma_{\nu} \gamma_{5} e_{2}^{c}\right)^{\dagger} \frac{1-P_{e_{1} e_{2}}}{2} \\
=\frac{1}{8}\left[f_{66}^{(0)}+f_{66}^{(1)}\left(\hat{\mathbf{p}}_{1} \cdot \hat{\mathbf{p}}_{2}\right)\right] \quad(\mu, \nu=0), \\
\sum_{s_{1}, s_{2}}\left(\bar{e}_{1} \gamma_{\mu} \gamma_{5} e_{2}^{c}\right)\left(\bar{e}_{1}\left(1 \pm \gamma_{5}\right) e_{2}^{c}\right)^{\dagger} \frac{1-P_{e_{1} e_{2}}}{2} \\
=\mp \frac{1}{2} f_{16}^{(0)} \quad(\mu=0) .
\end{gathered}
$$

Here, $\hat{\mathbf{p}}_{1} \cdot \hat{\mathbf{p}}_{2}=\cos \theta$ is the scalar product between the asymptotic momentum vectors of the two electrons, yielding the opening angle $0 \leq \theta \leq \pi$. In Eq. (86), if both the involved scalar currents are left-handed, the same result as for two right-handed currents holds. The quantities $f_{i j}^{(0)}=$ $f_{i j}^{(0)}\left(E_{1}, E_{2}\right)$ and $f_{i j}^{(1)}=f_{i j}^{(1)}\left(E_{1}, E_{2}\right)$ are given by

$f_{11}^{(0)}=\left|f^{-1-1}\right|^{2}+\left|f_{11}\right|^{2}+\left|f^{-1}{ }_{1}\right|^{2}+\left|f_{1}{ }^{-1}\right|^{2}$,

$f_{11 \pm}^{(1)}=-2\left[f^{-1}{ }_{1} f_{1}{ }^{-1} \pm f^{-1-1} f_{11}\right]$,

$f_{66}^{(0)}=16\left[\left|f^{-1-1}\right|^{2}+\left|f_{11}\right|^{2}\right], \quad f_{66}^{(1)}=32\left[f^{-1-1} f_{11}\right]$,

$f_{16}^{(0)}=4\left[\left|f_{11}\right|^{2}-\left|f^{-1-1}\right|^{2}\right], \quad f_{16}^{(1)}=0$.

We note that our results agree with those of Päs et al. [13] and Tomoda [48], except for the extra interference term $f_{11-}^{(1)}$ in Eq. (89) between the left- and right-handed scalar electron currents and the fact that these authors use the notation of Doi $[46,47]$, while we have used that of Tomoda [48]. The phase-space factors corresponding to $\mu=j$ or $\nu=j$ in Eqs. (87) and (88) are not shown, as their corresponding contributions to $0 \nu \beta \beta$ decay do not trigger a $0^{+} \rightarrow 0^{+}$transition, in the case of $S_{1 / 2}-S_{1 / 2}$ approximation, we are interested in (although they are relevant when general $0^{+} \rightarrow J^{+}$transitions are considered). All the above listed phase-space factors are given in terms of the underlying energy-dependent wave functions of the two electrons

$$
\begin{gathered}
f^{-1-1}=g_{-1}\left(E_{1}\right) g_{-1}\left(E_{2}\right), \\
f_{11}=f_{1}\left(E_{1}\right) f_{1}\left(E_{2}\right), \\
f^{-1}{ }_{1}=g_{-1}\left(E_{1}\right) f_{1}\left(E_{2}\right), \\
f_{1}^{-1}=f_{1}\left(E_{1}\right) g_{-1}\left(E_{2}\right) .
\end{gathered}
$$

\section{NUMERICAL RESULTS}

\section{A. Decay half-life and angular correlation}

Combining the above results, the coefficients $a\left(E_{1}\right)$ and $b\left(E_{1}\right)$ in the fully differential rate Eq. (17) for $0^{+} \rightarrow 0^{+}$ $0 \nu \beta \beta$ decay are given, respectively, by

$$
\begin{aligned}
a\left(E_{1}\right)= & 2 f_{11}^{(0)}\left|\sum_{I=1}^{3} \epsilon_{I} \mathcal{M}_{I}\right|^{2}+\frac{1}{8} f_{66}^{(0)}\left|\sum_{I=4}^{5} \epsilon_{I} \mathcal{M}_{I}\right|^{2} \\
& \mp f_{16}^{(0)} \operatorname{Re}\left[\left(\sum_{I=1}^{3} \epsilon_{I} \mathcal{M}_{I}\right)\left(\sum_{I=4}^{5} \epsilon_{I} \mathcal{M}_{I}\right)^{*}\right], \\
b\left(E_{1}\right)= & 2 f_{11 \pm}^{(1)}\left|\sum_{I=1}^{3} \epsilon_{I} \mathcal{M}_{I}\right|_{ \pm}^{2}+\frac{1}{8} f_{66}^{(1)}\left|\sum_{I=4}^{5} \epsilon_{I} \mathcal{M}_{I}\right|^{2} .
\end{aligned}
$$

They are expressed in terms of the NMEs in Eqs. (44)-(48) and the PSFs in Eqs. (89)-(91), where the summations as indicated are over the different current types $i=1,2,3,4,5$ including their different chiralities, $I=(i, X Y Z)$ with $X, Y$, $Z \in\{L, R\}$. In Eq. (96), the sign in front of $f_{16}^{(0)}$ is negative (positive) if the chirality of the electron scalar current involved in the interference term is $R(L)$. In Eq. (97), the \pm in the subscript of the norm symbolically denotes that the terms containing $\epsilon_{I}$ and $\epsilon_{J}^{*}$ corresponding to the same electron chiralities are accompanied by $f_{11+}^{(1)}$, while, for terms with opposite electron chiralities, the respective PSF is given by $f_{11 \text { - }}^{(1)}$ The contribution of the interference term between currents $i=1,2,3$ and $i=4,5$ vanishes in $b\left(E_{1}\right)$ due to $f_{16}^{(1)}=0$ in Eq. (91).

The basic nuclear matrix elements $\mathcal{M}_{F}, \mathcal{M}_{G T}$, and $\mathcal{M}_{T}$ on which the NMEs $\mathcal{M}_{I}$ are based are given in Table II for selected nuclei. The values are taken from Table IV of Ref. [32]. These matrix elements are given in dimensionless units; that is, they are multiplied by the massnumber-dependent radius $R_{A}=R_{0} A^{1 / 3}$ of the nucleus, where $R_{0}=1.2 \mathrm{fm}$.

We numerically calculate the electron wave functions according to Ref. [61] and as described in the previous section. Combining Eqs. (18), (19), (96), and (97), we then determine the single electron distribution $d \Gamma / d E_{1}$ and the 
TABLE II. Nuclear matrix elements $\mathcal{M}_{F}, \mathcal{M}_{G T}$, and $\mathcal{M}_{T}$ for selected nuclei, adopted from Ref. [32].

\begin{tabular}{llrr}
\hline \hline & $\mathcal{M}_{F}$ & $\mathcal{M}_{G T}$ & \multicolumn{1}{c}{$\mathcal{M}_{T}$} \\
\hline${ }^{76} \mathrm{Ge}$ & -42.8 & 104.0 & -26.9 \\
${ }^{82} \mathrm{Se}$ & -37.1 & 87.2 & -27.3 \\
${ }^{100} \mathrm{Mo}$ & -46.8 & 111.0 & 24.2 \\
${ }^{130} \mathrm{Te}$ & -37.9 & 84.8 & -16.6 \\
${ }^{136} \mathrm{Xe}$ & -29.7 & 66.8 & -12.7 \\
\hline \hline
\end{tabular}
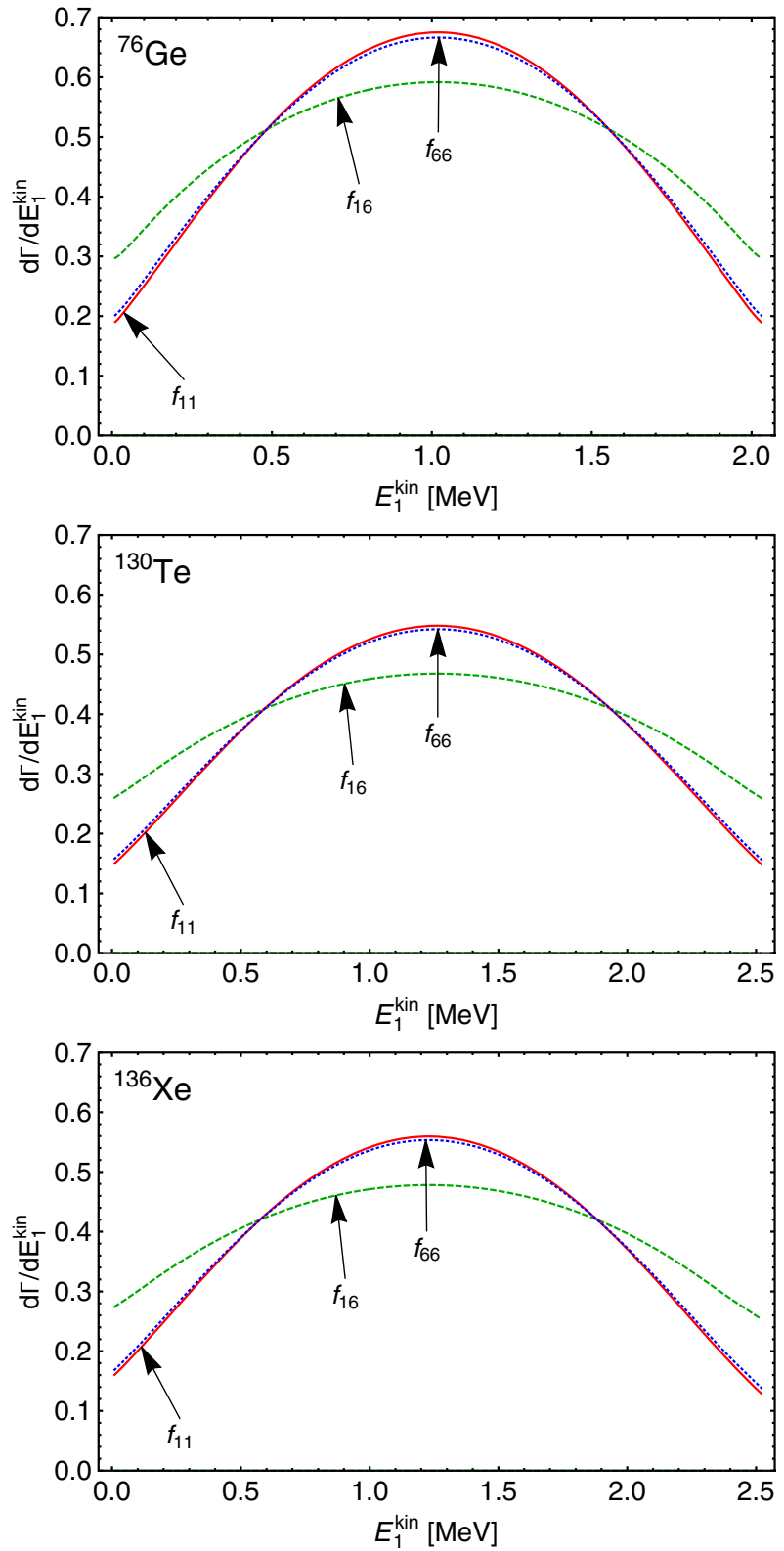

angular correlation $\alpha\left(E_{1}\right)$ for the three relevant phase-space factors that can occur under the presence of short-range operators: $f_{11}^{(a)}$ (for mechanisms $i=1,2,3$ with a scalar electron current), $f_{66}^{(a)}$ (for mechanisms $i=4,5$ with an axial-vector electron current), and $f_{16}^{(0)}$ (for interference between the two classes). In the latter case, the contribution $f_{16}^{(1)}$ to the angular coefficient $b\left(E_{1}\right)$ vanishes identically. The electron phase-space distribution $f_{11}$ is identical to that of the standard mass mechanism, calculated in the closure approximation. The results are shown in Fig. 2 for the $0 \nu \beta \beta$
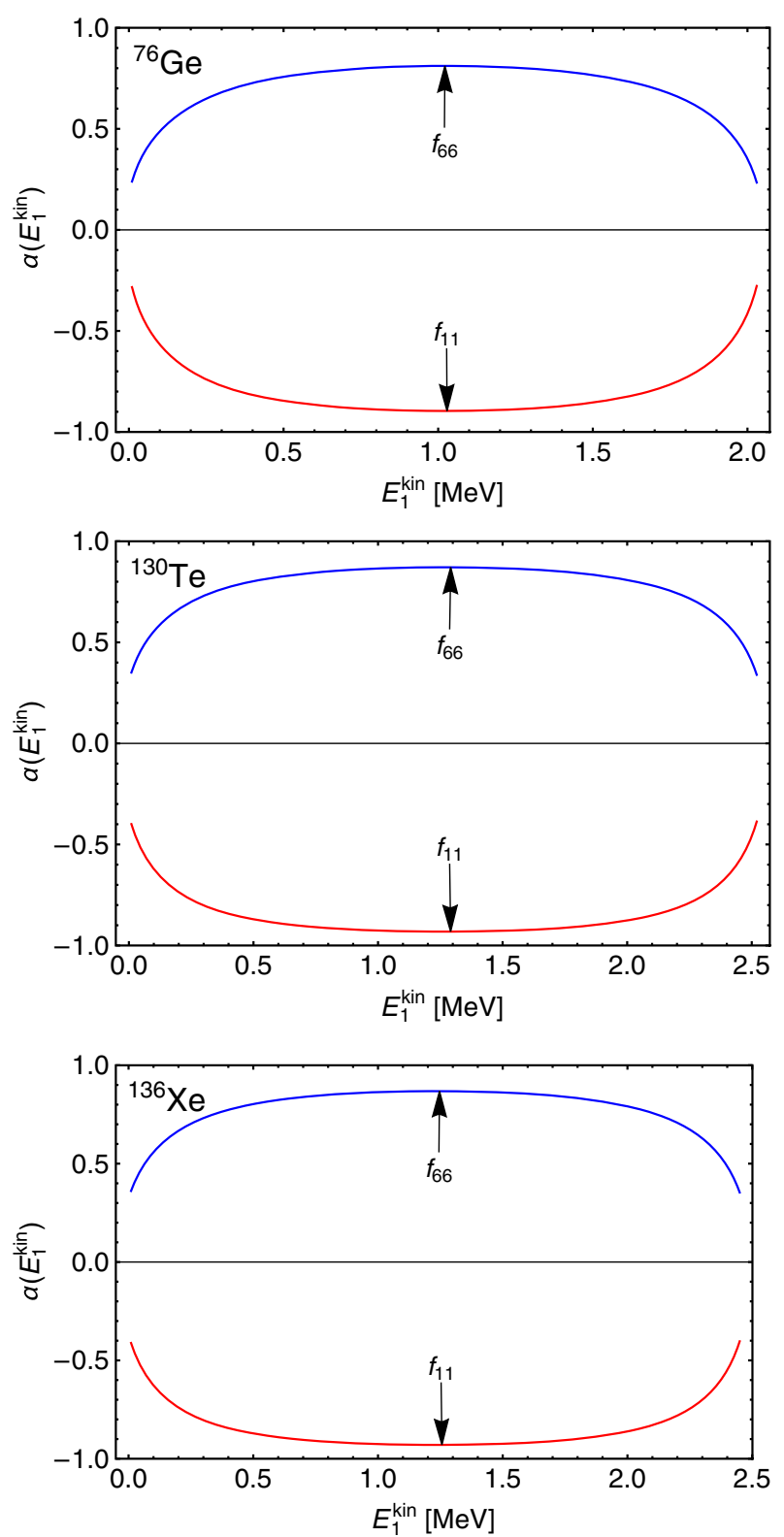

FIG. 2. Left panel: Single electron energy distribution $d \Gamma / d E_{1}^{\mathrm{kin}}$ as a function of the kinetic energy $E_{1}^{\mathrm{kin}}=E_{1}-m_{e}$ for the three different phase-space factors in Eq. (96), namely, $f_{11}, f_{66}$, and $f_{16}$. Right panel: Energy-dependent angular correlation $\alpha\left(E_{1}^{\text {kin }}\right)$ between the two electrons as a function of the kinetic energy $E_{1}^{\mathrm{kin}}$ for the phase-space factors $f_{11}$ and $f_{66}$ (identically zero for $f_{16}$ ). From top to bottom, the plots show the results for the three $0 \nu \beta \beta$ decay isotopes ${ }^{76} \mathrm{Ge},{ }^{130} \mathrm{Te}$, and ${ }^{136} \mathrm{Xe}$. 
decay isotopes ${ }^{76} \mathrm{Ge},{ }^{130} \mathrm{Te}$, and ${ }^{136} \mathrm{Xe}$. We plot both the normalized single energy distributions and the angular correlation as functions of the kinetic energy $E_{1}^{\mathrm{kin}}=E_{1}-$ $m_{e}$ of one of the electrons; i.e., the range is from zero up to the $Q_{\beta \beta}$ value of the respective isotope.

In all scenarios, the single energy distribution $d \Gamma / d E_{1}^{\mathrm{kin}}$ is of a hill-like shape; i.e., the two electrons preferably share the available kinetic energy equally. There is only a small difference between the $f_{11}$ and $f_{66}$ case, with the latter having a slightly flatter profile, that is unlikely to be distinguishable experimentally. The term $f_{16}$, corresponding to an interference between $i=1,2,3$ and $i=4,5$ mechanisms, has a more significantly flatter profile.

The angular correlation $\alpha\left(E_{1}^{\text {kin }}\right)$ is negative for $f_{11}$ and positive for $f_{66}$; i.e., in the former case, the electrons are preferably emitted back to back, whereas in the latter case they preferably fly in a similar direction. This allows one to potentially distinguish the scenarios resulting in $f_{66}$ from the standard mass mechanism as well as from scenarios corresponding to $f_{11}$.

Following Ref. [61], we define the integrated PSFs

$G_{i j}^{(a)}=\frac{2 C}{\ln 2} \frac{g_{i j}^{(a)}}{4 R_{A}^{2}} \int_{m_{e}}^{Q_{\beta \beta}+m_{e}} d E_{1} w\left(E_{1}\right) f_{i j}^{(a)}\left(E_{1}, Q_{\beta \beta}+2 m_{e}-E_{1}\right)$,

with $g_{11}^{(0)}=2, g_{11}^{(1)}=2, g_{66}^{(0)}=1 / 8, g_{66}^{(1)}=1 / 8, g_{16}^{(0)}=1$, and $g_{16}^{(1)}=0$ as well as $C$ and $w\left(E_{1}\right)$ as defined in Eqs. (18) and (19), respectively. The factor $1 / R_{A}^{2}$ has been introduced in Eq. (98) to conform with standard notation to compensate for the corresponding factor in the NMEs as discussed above. The numerical values of the PSFs $G_{i j}^{(a)}$, calculated in analogy with Ref. [61], are given in Table III. As mentioned before, the PSF $G_{16}^{(1)}$ vanishes identically; in addition, $G_{11-}^{(1)}$, corresponding to the interference between a right-handed and left-handed scalar electron current, is also zero as indicated in Table III.

With the above PSFs, the inverse $0 \nu \beta \beta$ decay half-life is then given by

$$
\begin{aligned}
T_{1 / 2}^{-1}= & G_{11}^{(0)}\left|\sum_{I=1}^{3} \epsilon_{I} \mathcal{M}_{I}\right|^{2}+G_{66}^{(0)}\left|\sum_{I=4}^{5} \epsilon_{I} \mathcal{M}_{I}\right|^{2} \\
& \mp G_{16}^{(0)} \operatorname{Re}\left[\left(\sum_{I=1}^{3} \epsilon_{I} \mathcal{M}_{I}\right)\left(\sum_{I=4}^{5} \epsilon_{I} \mathcal{M}_{I}\right)^{*}\right] .
\end{aligned}
$$

Analogous to Eq. (96), the sign in front of $G_{16}^{(0)}$ is negative (positive) if the chirality of the electron scalar current involved in the interference term is $R(L)$.

We can also calculate the integrated angular correlation factors as

$$
K_{j k}=\frac{B}{A}=\frac{G_{j k}^{(1)}}{G_{j k}^{(0)}},
$$

in the three different cases $j k=11,66$, and 16. The numerical values of $K_{11}$ and $K_{66}$ are also listed in Table III, whereas $K_{16}=0$ is always identically zero. As already discussed, in view of the opposite sign for 11 and 66 in Table III, an eventual measurement of the angular correlation will allow a discrimination of the two types of nonstandard mechanisms.

\section{B. Bounds on couplings}

In principle, a given underlying particle physics model may give rise to several contributions, and/or mixing among the corresponding Wilson coefficients will induce contributions through radiative effects from the scale of new physics, through the electroweak scale and down to the scale of QCD. The above formulas for the decay rate take into account all possible short-range contributions where the $\epsilon_{I}$ factors are to be understood to be effective at the QCD scale. To determine the numerical sensitivity to the $\epsilon_{I}$ factors, we here make the commonly considered simplifying assumption that only one term $\epsilon_{I}$ is different from zero and, thus, only one mechanism contributes at a time.

The resulting upper limits on the $\epsilon_{I}$ factors are estimated in Table IV, based on the above calculation of the $0 \nu \beta \beta$ halflife and using the most stringent experimental bounds for the isotopes ${ }^{76} \mathrm{Ge}$ [75], ${ }^{130} \mathrm{Te}$ [76], and ${ }^{136} \mathrm{Xe}$ [77]. In

TABLE III. Phase-space factors $G_{j k}^{(i)}$ for selected nuclei, calculated in analogy with Ref. [61], and corresponding angular correlation factors $K_{11}$ and $K_{66}\left(K_{16}=0\right.$ is always identically zero). All PSFs are given in units of $10^{-15} \mathrm{yr}^{-1}$ as indicated.

\begin{tabular}{lccccccccc}
\hline \hline$G_{j k}^{(i)}\left[10^{-15} \mathrm{yr}^{-1}\right]$ & $G_{11}^{(0)}$ & $G_{66}^{(0)}$ & $G_{16}^{(0)}$ & $G_{11+}^{(1)}$ & $G_{11-}^{(1)}$ & $G_{66}^{(1)}$ & $G_{16}^{(1)}$ & $K_{11}$ & $K_{66}$ \\
\hline${ }^{76} \mathrm{Ge}$ & 4.72 & 2.64 & 1.7 & -3.90 & 0 & 1.95 & 0 & -0.83 & 0.74 \\
${ }^{82} \mathrm{Se}$ & 20.4 & 10.8 & 5.9 & -18.1 & 0 & 9.10 & 0 & -0.89 & 0.84 \\
${ }^{100} \mathrm{Mo}$ & 31.8 & 17.0 & 8.4 & -28.6 & 0 & 14.2 & 0 & -0.90 & 0.84 \\
${ }^{130} \mathrm{Te}$ & 28.4 & 15.3 & 8.7 & -24.8 & 0 & 12.4 & 0 & -0.87 & 0.81 \\
${ }^{136} \mathrm{Xe}$ & 29.2 & 15.7 & 9.0 & -25.4 & 0 & 12.7 & 0 & -0.87 & 0.81 \\
\hline \hline
\end{tabular}


TABLE IV. Estimates of upper limits on the absolute values of the $\epsilon_{I}$ couplings (in units of $10^{-10}$ ) from current experimental bounds, assuming only one contribution is different from zero at a time. The chiralities of the involved quark currents are specified, as the corresponding bounds differ. The label $X X$ stands for the case when both chiralities are the same, i.e., $X X=R R$ or $X X=L L$. The experimental bounds at $90 \%$ confidence level, reported by recent searches at KamLAND-Zen [77], GERDA [75], and CUORE [76] are included.

\begin{tabular}{lcccccccrr}
\hline \hline & $T_{1 / 2}^{\exp }[y]$ & $\left|\epsilon_{1}^{X X}\right|$ & $\left|\epsilon_{1}^{L R}\right|$ & $\left|\epsilon_{2}^{X X}\right|$ & $\left|\epsilon_{3}^{X X}\right|$ & $\left|\epsilon_{3}^{L R}\right|$ & $\left|\epsilon_{4}^{X X, L R}\right|$ & $\left|\epsilon_{5}^{X X}\right|$ & $\left|\epsilon_{5}^{R L, L R}\right|$ \\
\hline${ }^{76} \mathrm{Ge}$ & $5.3 \times 10^{25}[75]$ & 1.5 & 1.5 & 190 & 110 & 220 & 250 & 60 & 50 \\
${ }^{130} \mathrm{Te}$ & $2.8 \times 10^{24}[76]$ & 3.5 & 3.4 & 420 & 240 & 490 & 550 & 140 & 120 \\
${ }^{136} \mathrm{Xe}$ & $1.1 \times 10^{26}[77]$ & 0.57 & 0.57 & 84 & 50 & 110 & 110 & 23 & 19 \\
\hline \hline
\end{tabular}

TABLE V. Sensitivity estimates on the absolute values of the $\epsilon_{I}$ couplings (in units of $10^{-10}$ ) from a prospective future experimental sensitivity $T_{1 / 2}^{\text {future }}=10^{27} \mathrm{yr}$, assuming only one contribution is different from zero at a time. The label $X X$ stands for the case when both chiralities are the same, i.e., $X X=R R$ or $X X=L L$.

\begin{tabular}{lcllcccccc}
\hline \hline & $T_{1 / 2}^{\exp }[y]$ & $\left|\epsilon_{1}^{X X}\right|$ & $\left|\epsilon_{1}^{L R}\right|$ & $\left|\epsilon_{2}^{X X}\right|$ & $\left|\epsilon_{3}^{X X}\right|$ & $\left|\epsilon_{3}^{L R}\right|$ & $\left|\epsilon_{4}^{X X, L R}\right|$ & $\left|\epsilon_{5}^{X X}\right|$ & $\left|\epsilon_{5}^{R L, L R}\right|$ \\
\hline${ }^{76} \mathrm{Ge}$ & $10^{27}$ & 0.35 & 0.35 & 44 & 26 & 50 & 58 & 14 & 11 \\
${ }^{82} \mathrm{Se}$ & $10^{27}$ & 0.19 & 0.19 & 25 & 15 & 30 & 34 & 7.8 & 6.5 \\
${ }^{100} \mathrm{Mo}$ & $10^{27}$ & 0.2 & 0.2 & 16 & 8.9 & 16 & 20 & 8.7 & 6.5 \\
${ }^{130} \mathrm{Te}$ & $10^{27}$ & 0.18 & 0.18 & 22 & 13 & 26 & 29 & 7.5 & 6.1 \\
${ }^{136} \mathrm{Xe}$ & $10^{27}$ & 0.19 & 0.19 & 28 & 17 & 35 & 38 & 7.6 & 6.4 \\
\hline \hline
\end{tabular}

Table V, we then show the estimated reach with respect to the effective couplings assuming a common future experimental sensitivity of $T_{1 / 2}^{\text {future }}=10^{27} \mathrm{yr}$ including two additional potentially interesting isotopes, ${ }^{82} \mathrm{Se}$ and ${ }^{100} \mathrm{Mo}$. In both tables, upper limits on the absolute values $\left|\epsilon_{i}^{X Y}\right|$ of the effective couplings are shown. Different chiralities of the quark currents in the operators lead to different bounds as indicated; here $\epsilon_{i}^{X X}$ denotes the case where the chiralities of the two quark currents are equal, i.e., $X X=L L$ and $X X=R R$. For $\epsilon_{2}$ and $\epsilon_{4}$, the bounds do not depend on the choice of chiralities.

We would like to stress that, in constructing these tables, we have used the values of $\mathcal{M}_{F}, \mathcal{M}_{G T}$, and $\mathcal{M}_{T}$ given in Table II and we estimate the values of the other NMEs involved by replacing $\left(\mathbf{q} / m_{p}\right)^{2}=0.01$ as a rough average and by neglecting the effect of differently shaped $\mathbf{q}^{2}$ dependence of form factors. We do not attempt to assess the resulting uncertainty, and more accurate results based on the actual calculation of the additional NMEs will be reported in a future work.

\section{QCD running of couplings}

The above limits on the effective couplings of exotic shortrange mechanisms have been so far implicitly assumed to apply at the QCD scale $\Lambda_{\mathrm{QCD}} \approx 1 \mathrm{GeV}$ with one coupling set different from zero. Following Ref. [78], it is possible to be more accurate and assume each of these couplings to exist at a certain new physics scale $\Lambda_{\mathrm{NP}} \approx 1 \mathrm{TeV}$ and run it down to
$\Lambda_{\mathrm{QCD}}$, where the appropriate bound can be set employing the experimental limit on $0 \nu \beta \beta$ decay half-life. Consequently, the values obtained in this way can be compared with constraints derived from collider experiments.

The renormalization group equation (RGE) for a set of coupled Wilson coefficients $\mathbf{c}=\left(c_{1}, c_{2}, \ldots, c_{n}\right)^{T}$ reads

$$
\frac{d \mathbf{c}(\mu)}{d \log \mu}=\gamma^{T} \cdot \mathbf{c}(\mu)
$$

where $\gamma$ is the anomalous dimension matrix in the $\overline{\mathrm{MS}}$ scheme. At one-loop order, it is given by $\gamma=-2\left(b-2 C_{F} \mathbb{I}\right)$, with $C_{F}$ being the color factor and $b$ a $\mu$-independent constant matrix. The solution to Eq. (101) is most conveniently written in matrix form:

$$
\mathbf{c}(\mu)=U\left(\mu, \Lambda_{\mathrm{NP}}\right) \cdot \mathbf{c}\left(\Lambda_{\mathrm{NP}}\right)
$$

where $\mu$ and $\Lambda_{\mathrm{NP}}$ are the low- and high-energy scales, respectively, between which the coefficients are evolved.

We apply the procedure sketched above to the case of the effective couplings $c_{I} \equiv \epsilon_{I}(1 \mathrm{TeV})$ of short-range operators triggering $0 \nu \beta \beta$ decay at the scale $\Lambda_{\mathrm{NP}}=1 \mathrm{TeV}$. In this case, the evolution matrix $U=U\left(\Lambda_{\mathrm{QCD}}, \Lambda_{\mathrm{NP}}\right)$ of Wilson coefficients between $\Lambda_{\mathrm{NP}}$ and $\Lambda_{\mathrm{QCD}}$ is rather sparse; its only nonzero elements read [78] 
TABLE VI. As in Table IV but for the effective couplings defined at the average new physics scale $\Lambda_{\mathrm{NP}}=1 \mathrm{TeV}$.

\begin{tabular}{lcccccccccc}
\hline \hline & $T_{1 / 2}^{\exp }[y]$ & $\left|c_{1}^{X X}\right|$ & $\left|c_{1}^{L R}\right|$ & $\left|c_{2}^{X X}\right|$ & $\left|c_{3}^{X X}\right|$ & $\left|c_{3}^{L R}\right|$ & $\left|c_{4}^{X X}\right|$ & $\left|c_{4}^{L R}\right|$ & $\left|c_{5}^{X X}\right|$ & $\left|c_{5}^{R L, L R}\right|$ \\
\hline${ }^{76} \mathrm{Ge}$ & $5.3 \times 10^{25}[75]$ & 0.62 & 0.36 & 88 & 160 & 260 & 580 & 400 & 25 & 12 \\
${ }^{130} \mathrm{Te}$ & $2.8 \times 10^{24}[76]$ & 1.4 & 0.83 & 200 & 350 & 580 & 1300 & 880 & 59 & 28 \\
${ }^{136} \mathrm{Xe}$ & $1.1 \times 10^{26}[77]$ & 0.24 & 0.14 & 32 & 72 & 130 & 250 & 190 & 9.6 & 4.7 \\
\hline \hline
\end{tabular}

$$
\begin{aligned}
U_{(12)}^{X X} & =\left(\begin{array}{cc}
2.39 & 0.02 \\
-3.83 & 0.35
\end{array}\right), \quad U_{(31)}^{L R}=\left(\begin{array}{cc}
0.84 & -2.19 \\
0 & 4.13
\end{array}\right), \\
U_{(45)}^{X X} & =\left(\begin{array}{cc}
0.35 & -0.96 i \\
-0.06 i & 2.39
\end{array}\right), \\
U_{(3)}^{X X} & =0.70, \quad U_{(4)}^{L R}=0.62, \quad U_{(5)}^{L R}=4.13, \quad(103)
\end{aligned}
$$

where the subscripts denote the respective short-range operator(s) and the superscripts the chiralities of the quark currents involved. For example, the matrix $U_{(12)}^{X X}$ describes the mixing between the first and second short-range operators involving quark currents with the same chiralities. Using Eq. (103) and the approximated values of NMEs Eqs. (44)-(48), the corresponding bounds on couplings $c_{I}$ are obtained and shown in Table VI. Analogous to Table IV, we take only one effective coupling different from zero at a time. The difference is that we make this assumption now at the scale $\Lambda_{\mathrm{NP}}$ and we use the above Wilson RGE solution to evolve the couplings to $\Lambda_{\mathrm{QCD}}$ to calculate the $0 \nu \beta \beta$ decay rate, potentially with more than one coupling active due to mixing.

The resulting bounds on the couplings $\left|c_{I}\right|$ at $\Lambda_{\mathrm{NP}}$, including QCD running effects, displayed in Table VI are weaker or more stringent than those in Table IV, depending on the operator in question. It should be noted that the limit on $\left|\epsilon_{4}\right|$ splits into two different values $c_{4}^{X X}$ and $c_{4}^{L R}$, since the running depends on the quark current chiralities. As for the effects of operator mixing, the limits on $\left|c_{4}\right|$ are, for example, less stringent due to the size of the corresponding RG evolution matrix elements, which are always smaller than 1 . In the case of the mixing between $\mathcal{O}_{1}^{X X}$ and $\mathcal{O}_{2}^{X X}$, the limit on $\left|c_{2}^{X X}\right|$ is not much affected by the mixing, because the relevant element of the evolution matrix is small, $\left[U_{(12)}^{X X}\right]_{12}=0.02$; hence, the expectedly strong contribution from $\mathcal{O}_{1}^{X X}$ (large NMEs) to $c_{2}^{X X}$ is suppressed. As a result, the bounds in Table VI are not drastically different from those in Table IV, despite the strong variation in sensitivity to the couplings $\epsilon_{i}$.

\section{SUMMARY AND CONCLUSION}

In this article, we have developed a general formalism for short-range mechanisms contributing to neutrinoless double beta decay in an effective operator approach. Such contributions will arise when the lepton number is broken at a new physics scale $\Lambda_{\mathrm{NP}}$ much larger than the typical energy scale of $0 \nu \beta \beta$ decay $q \approx 100 \mathrm{MeV}$. We have calculated the expected $0 \nu \beta \beta$ half-lives by making use of the phase-space factors calculated in the same way, as described in Ref. [61] and the leading-order nuclear matrix elements of Ref. [32], where we especially elucidate the different contributions arising from general form factors in the nucleon currents. Unlike, for example, Ref. [13], we calculate all terms of the order of $\mathbf{q}^{2} / m_{p}^{2}$ in the nonrelativistic expansion of the combined nucleon currents. Some of these terms play an important role, as, e.g., the pseudoscalar terms which are enhanced by a large value of $g_{P S}$ or $g_{P}$. This consequently results in additional NMEs in Eqs. (44)-(48) not accounted for in Ref. [13]. Furthermore, we also evaluate new phase-space factors originating from the electron currents, including interference effects of different short-range contributions. The results of the present paper complement those of Ali, Borisov, and Zhuridov [50] for long-range neutrinoless double beta decay. We also find that the angular correlation between the two emitted electrons is different for different mechanisms, although in our case there are only two types of angular correlations, one for terms 1,2, and 3 and one for terms 4 and 5 of the effective Lagrangian.

Using experimental bounds on half-lives and estimating the novel matrix elements arising in short-range contributions, we have calculated the numerical limits on the effective new physics parameters $\epsilon_{I}$. To leading order, only the standard Fermi, Gamow-Teller matrix elements appear, but especially the enhanced values of the exotic and induced pseudoscalar couplings in the form factor approach necessitate the inclusion of higher-order terms in $\mathbf{q} / m_{p}$. This then requires the determination of different nuclear matrix elements $\mathcal{M}_{F}^{\prime}, \mathcal{M}_{G T}^{\prime}, \mathcal{M}_{T}^{\prime}, \mathcal{M}_{F}^{\prime \prime}, \mathcal{M}_{G T}^{\prime \prime}$, and $\mathcal{M}_{T}^{\prime \prime}$, the calculation of which is currently under way and will be presented in a subsequent publication. They are crucially important to accurately determine dominant contributions to short-range $0 \nu \beta \beta$ decay and to verify the strong limits we obtain on the effective new physics parameters ranging between $\epsilon_{I} \approx 10^{-10}$ and $10^{-7}$, which correspond to new physics scales in the multi-TeV region. Short-range contributions scale as $\epsilon \propto 1 / \Lambda_{\mathrm{NP}}^{5}$, and thus an increase in sensitivity on $\epsilon$ by an order of magnitude will improve a limit on $\Lambda_{\mathrm{NP}}$ only by a factor of $\approx 1.6$. Nevertheless, accurate calculations of the limits and sensitivities are crucially important, as they probe the phenomenologically interesting TeV scale. A robust map of potential sources of lepton number violation in this energy region will help us to 
get a better understanding of the mechanism of neutrino mass generation.

\section{ACKNOWLEDGMENTS}

This work was supported in part by the U.S. Department of Energy (Grant No. DE-FG-02-91ER-40608) and the United Kingdom Royal Society International Exchange program. The work of J. K. was supported by the Väisälä Foundation and the Academy of Finland (Grant No. 314733). The work of L. G. and F. F. D. was supported by a United Kingdom Science and Technology Facilities Council Consolidated Grant. L. G. and F. F. D. thank Martin Hirsch for useful discussions and support on the QCD renormalization running, as well as Amr Hossameldin for carefully checking the nucleon current products.

Note added.—Recently, we noticed the preprint [79], which discusses short-range contributions as well but using a complementary approach based on the chiral effective field theory.

\section{APPENDIX A: NUCLEON CURRENT PRODUCTS}

In the following, we explicitly show the products of the nonrelativistically approximated hadronic currents for each of the five terms of the effective short-range Lagrangian Eq. (5). Generally, we include all the terms up to linear order in $\mathbf{q} / m_{p}$; from higher orders, only terms enhanced by large form factors $F_{P S}$ and/or $F_{P}$ are kept. All the products are symmetrized in indices $a \leftrightarrow b$ labeling individual nucleons in the nuclei. In the case of each term, we keep track of signs corresponding to different combinations of chiralities, and these signs we show as a row vector in front of every single term of the expressions. In the case of the first three products of hadronic currents (i.e., those proportional to $\epsilon_{1}, \epsilon_{2}$, and $\epsilon_{3}$ ), three possibilities are presented, and they correspond to the following combinations of chiralities (in this ordering): $R R, L L$, and $(1 / 2)(R L+L R)$. For the fourth and fifth products (proportional to $\epsilon_{4}$ and $\epsilon_{5}$ ), a row of four signs is shown, as in those cases the two hadronic currents have different Lorentz structures; thus, all four possible combinations of chiralities have to be considered (in this ordering): $R R, L L$, $R L$, and $L R$.

Term 1: $J J j$.- The product of currents $J J$ is

$$
\begin{aligned}
\Pi_{1} & \equiv \frac{1}{2}\left[J_{\circ, a} J_{\circ, b}+J_{\circ, b} J_{\circ, a}\right] \\
& =(+++) F_{S}^{2}\left(q^{2}\right) I_{a} I_{b} \quad[\mathcal{O}(1) S-S] \\
& (++-) \frac{F_{P S}^{2}\left(q^{2}\right)}{4 m_{p}^{2}}\left(\boldsymbol{\sigma}_{a} \cdot \mathbf{q}\right)\left(\boldsymbol{\sigma}_{b} \cdot \mathbf{q}\right)+\cdots, \quad[\mathcal{O}(100) S-S]
\end{aligned}
$$

where the term proportional to $F_{P S}^{2}\left(q^{2}\right)$ can be recoupled using the following relation:

$$
\left(\boldsymbol{\sigma}_{a} \cdot \mathbf{q}\right)\left(\boldsymbol{\sigma}_{b} \cdot \mathbf{q}\right)=\frac{1}{3}\left(\boldsymbol{\sigma}_{a} \cdot \boldsymbol{\sigma}_{b}\right) \mathbf{q}^{2}-\frac{1}{3}\left[\mathbf{q}^{2}-\frac{1}{3}\left(\mathbf{q} \cdot \hat{\mathbf{r}}_{a b}\right)^{2}\right] \mathbf{S}_{a b},
$$

with $\mathbf{S}_{a b}=3\left(\boldsymbol{\sigma}_{a} \cdot \hat{\mathbf{r}}_{a b}\right)\left(\boldsymbol{\sigma}_{b} \cdot \hat{\mathbf{r}}_{a b}\right)-\left(\boldsymbol{\sigma}_{a} \cdot \boldsymbol{\sigma}_{b}\right)$.

Term 2: $J^{\mu \nu} J_{\mu \nu} j$.- - For the second term of the short-range part of the Lagrangian, we get the following approximation of the nuclear currents:

$$
\begin{aligned}
\Pi_{2} & \equiv \frac{1}{2}\left[J_{\circ, a}^{\mu \nu} J_{\circ \mu \nu, b}+J_{\circ, b}^{\mu \nu} J_{\circ \mu \nu, a}\right] \\
& =(---) 2 F_{T_{1}}^{2}\left(q^{2}\right)\left(\boldsymbol{\sigma}_{a} \cdot \boldsymbol{\sigma}_{b}\right)+\cdots \quad[\mathcal{O}(1) S-S]
\end{aligned}
$$

Term 3: $J^{\mu} J_{\mu} j$.- Approximating the nuclear currents for the third term, we obtain

$$
\begin{aligned}
& \Pi_{3} \equiv \frac{1}{2}\left[J_{\circ, a}^{\mu} J_{\circ \mu, b}+J_{\circ, b}^{\mu} J_{\circ \mu, a}\right] \\
& =(+++) F_{V}^{2}\left(q^{2}\right) I_{a} I_{b} \\
& (--+) F_{A}^{2}\left(q^{2}\right)\left(\boldsymbol{\sigma}_{a} \cdot \boldsymbol{\sigma}_{b}\right) \\
& (++-) 2 \frac{F_{A}\left(q^{2}\right) F_{P}\left(q^{2}\right)}{4 m_{p}^{2}}\left(\boldsymbol{\sigma}_{a} \cdot \mathbf{q}\right)\left(\boldsymbol{\sigma}_{b} \cdot \mathbf{q}\right) \\
& {[\mathcal{O}(1) S-S]} \\
& (+++) \frac{\left(F_{V}\left(q^{2}\right)+F_{W}\left(q^{2}\right)\right)^{2}}{4 m_{p}^{2}}\left(\boldsymbol{\sigma}_{a} \times \mathbf{q}\right)\left(\boldsymbol{\sigma}_{b} \times \mathbf{q}\right) \quad[\mathcal{O}(0.1) S-S] \\
& (--+) \frac{F_{P}^{2}\left(q^{2}\right)}{16 m_{p}^{4}} \mathbf{q}^{2}\left(\boldsymbol{\sigma}_{a} \cdot \mathbf{q}\right)\left(\boldsymbol{\sigma}_{b} \cdot \mathbf{q}\right)+\cdots, \quad[\mathcal{O}(1) S-S]
\end{aligned}
$$

where the term proportional to $\left(F_{V}\left(q^{2}\right)+F_{W}\left(q^{2}\right)\right)^{2}$ can be recoupled as follows: 


$$
\left(\boldsymbol{\sigma}_{a} \times \mathbf{q}\right)\left(\boldsymbol{\sigma}_{b} \times \mathbf{q}\right)=-\frac{1}{3}\left(\boldsymbol{\sigma}_{a} \cdot \boldsymbol{\sigma}_{b}\right) \mathbf{q}^{2}-\frac{1}{6}\left[\mathbf{q}^{2}-\frac{1}{3}\left(\mathbf{q} \cdot \hat{\mathbf{r}}_{a b}\right)^{2}\right] \mathbf{S}_{a b}
$$

and as before $\mathbf{S}_{a b}=3\left(\boldsymbol{\sigma}_{a} \cdot \hat{\mathbf{r}}_{a b}\right)\left(\boldsymbol{\sigma}_{b} \cdot \hat{\mathbf{r}}_{a b}\right)-\left(\boldsymbol{\sigma}_{a} \cdot \boldsymbol{\sigma}_{b}\right)$.

Term 4: $J^{\mu} J_{\mu \nu} j^{\nu}$.- The product of the tensor and vector nuclear current in the fourth term can be nonrelativistically approximated as

$$
\begin{aligned}
& \Pi_{4 \nu, a b} \equiv \frac{1}{2}\left[J_{\circ, a}^{\mu} J_{\circ \mu \nu, b}+J_{\circ, b}^{\mu} J_{\circ \mu \nu, a}\right] \\
& \approx g_{\nu}{ }^{0}\left\{(--++) i F_{A}\left(q^{2}\right) F_{T_{1}}\left(q^{2}\right)\left(\boldsymbol{\sigma}_{a} \cdot \boldsymbol{\sigma}_{b}\right)\right. \\
& \left.(++--) i \frac{F_{P}\left(q^{2}\right) F_{T_{1}}\left(q^{2}\right)}{4 m_{p}^{2}}\left(\boldsymbol{\sigma}_{a} \cdot \mathbf{q}\right)\left(\boldsymbol{\sigma}_{b} \cdot \mathbf{q}\right)\right\} \\
& +g_{\nu}{ }^{i}\left\{(+--+) \frac{i}{2} F_{V}\left(q^{2}\right) F_{T_{1}}\left(q^{2}\right)\left(I_{a} \sigma_{b i}+I_{b} \sigma_{a i}\right)\right. \\
& (----) i \frac{F_{V}\left(q^{2}\right)\left[F_{T_{1}}\left(q^{2}\right)-2 F_{T_{2}}\left(q^{2}\right)\right]}{2 m_{p}} q_{i} I_{a} I_{b} \\
& (----) \frac{F_{V}\left(q^{2}\right) F_{T_{1}}\left(q^{2}\right)}{4 m_{p}} \\
& \times\left[I_{a}\left(\boldsymbol{\sigma}_{b} \times \mathbf{Q}\right)_{i}+I_{b}\left(\boldsymbol{\sigma}_{a} \times \mathbf{Q}\right)_{i}\right] \\
& (----) \frac{F_{V}\left(q^{2}\right) F_{T_{1}}\left(q^{2}\right)}{4 m_{p}} \times\left[I_{a}\left(\boldsymbol{\sigma}_{b} \times \mathbf{Q}\right)_{i}+I_{b}\left(\boldsymbol{\sigma}_{a} \times \mathbf{Q}\right)_{i}\right] \\
& (----) i \frac{\left[F_{V}\left(q^{2}\right)+F_{W}\left(q^{2}\right)\right] F_{T_{1}}\left(q^{2}\right)}{4 m_{p}} \\
& \times\left[2 q_{i}\left(\boldsymbol{\sigma}_{a} \cdot \boldsymbol{\sigma}_{b}\right)-\sigma_{a i}\left(\mathbf{q} \cdot \boldsymbol{\sigma}_{b}\right)-\sigma_{b i}\left(\mathbf{q} \cdot \boldsymbol{\sigma}_{a}\right)\right] \\
& (--++) \frac{F_{A}\left(q^{2}\right) F_{T_{1}}\left(q^{2}\right)}{4 m_{p}}\left[\left(\boldsymbol{\sigma}_{a} \cdot \mathbf{Q}\right) \sigma_{b i}+\left(\boldsymbol{\sigma}_{b} \cdot \mathbf{Q}\right) \sigma_{a i}\right] \\
& (++--) \frac{F_{A}\left(q^{2}\right)\left[F_{T_{1}}\left(q^{2}\right)-2 F_{T_{2}}\left(q^{2}\right)\right]}{4 m_{p}} \\
& \times\left[\left(\boldsymbol{\sigma}_{a} \times \mathbf{q}\right)_{i} I_{b}+\left(\boldsymbol{\sigma}_{b} \times \mathbf{q}\right)_{i} I_{a}\right] \\
& (--++) i \frac{F_{A}\left(q^{2}\right) F_{T_{1}}\left(q^{2}\right)}{4 m_{p}} \\
& \times\left[\sigma_{a i}\left(\mathbf{Q} \cdot \boldsymbol{\sigma}_{b}\right)+\sigma_{b i}\left(\mathbf{Q} \cdot \boldsymbol{\sigma}_{a}\right)-2 Q_{i}\left(\boldsymbol{\sigma}_{a} \cdot \boldsymbol{\sigma}_{b}\right)\right] \\
& (--++) i \frac{F_{P}\left(q^{2}\right) F_{T_{1}}\left(q^{2}\right)}{8 m_{p}^{2}} q^{0} \times\left[\left(\boldsymbol{\sigma}_{a} \cdot \mathbf{q}\right) \sigma_{b i}+\left(\boldsymbol{\sigma}_{b} \cdot \mathbf{q}\right) \sigma_{a i}\right] \\
& (++--) \frac{F_{P}\left(q^{2}\right) F_{T_{1}}\left(q^{2}\right)}{16 m_{p}^{3}}\left[\sigma_{a i}(\mathbf{q} \cdot \mathbf{Q})\left(\mathbf{q} \cdot \boldsymbol{\sigma}_{b}\right)\right. \\
& \left.\left.+\sigma_{b i}(\mathbf{q} \cdot \mathbf{Q})\left(\mathbf{q} \cdot \boldsymbol{\sigma}_{a}\right)-2 Q_{i}\left(\mathbf{q} \cdot \boldsymbol{\sigma}_{b}\right)\left(\mathbf{q} \cdot \boldsymbol{\sigma}_{b}\right)\right]\right\}+\cdots \\
& {[\mathcal{O}(1) S-S]} \\
& {[\mathcal{O}(1) S-S]} \\
& {[\mathcal{O}(1) S-P]} \\
& {[\mathcal{O}(0.1) S-P]} \\
& {[\mathcal{O}(0.1) S-P]} \\
& {[\mathcal{O}(0.1) S-P]} \\
& {[\mathcal{O}(0.1) S-P]} \\
& {[\mathcal{O}(0.1) S-P]} \\
& {[\mathcal{O}(0.1) S-P]} \\
& {[\mathcal{O}(0.1) S-P]} \\
& {[\mathcal{O}(0.1) S-P]} \\
& {[\mathcal{O}(0.1) S-P]}
\end{aligned}
$$


Term 5: $J^{\mu} J j_{\mu}$ - -Approximating the nuclear currents in this case, we obtain

$$
\begin{aligned}
& \Pi_{5}^{\mu} \equiv \frac{1}{2}\left[J_{\circ, a}^{\mu} J_{\circ, b}+J_{\circ, b}^{\mu} J_{\circ, a}\right] \\
& \approx g^{\mu}\left\{(++++) F_{S}\left(q^{2}\right) F_{V}\left(q^{2}\right) I_{a} I_{b} \quad[\mathcal{O}(1) S-S]\right. \\
& (++--) \frac{F_{P S}\left(q^{2}\right) F_{A}\left(q^{2}\right)}{8 m_{p}^{2}}\left[\left(\boldsymbol{\sigma}_{a} \cdot \mathbf{Q}\right)\left(\boldsymbol{\sigma}_{b} \cdot \mathbf{q}\right)+\left(\boldsymbol{\sigma}_{a} \cdot \mathbf{q}\right)\left(\boldsymbol{\sigma}_{b} \cdot \mathbf{Q}\right)\right] \quad[\mathcal{O}(1) S-S] \\
& \begin{array}{ll}
\left.(--++) \frac{F_{P S}\left(q^{2}\right) F_{P}\left(q^{2}\right)}{8 m_{p}^{3}} q^{0}\left(\boldsymbol{\sigma}_{a} \cdot \mathbf{q}\right)\left(\boldsymbol{\sigma}_{b} \cdot \mathbf{q}\right)\right\} & {[\mathcal{O}(1) S-S]}
\end{array} \\
& +g^{\mu}{ }_{i}\left\{(-+-+) \frac{F_{S}\left(q^{2}\right) F_{A}\left(q^{2}\right)}{2}\left(\sigma_{a}^{i} I_{b}+\sigma_{b}^{i} I_{a}\right) \quad[\mathcal{O}(1) S-P]\right. \\
& \begin{array}{ll}
(----) \frac{F_{S}\left(q^{2}\right) F_{V}\left(q^{2}\right)}{2 m_{p}} Q^{i} I_{a} I_{b} & {[\mathcal{O}(0.1) S-P]}
\end{array} \\
& (++++) i \frac{F_{S}\left(q^{2}\right)\left[F_{V}\left(q^{2}\right)+F_{W}\left(q^{2}\right)\right]}{4 m_{p}} \\
& \times\left[\left(\boldsymbol{\sigma}_{a} \times \mathbf{q}\right)^{i} I_{b}+\left(\boldsymbol{\sigma}_{b} \times \mathbf{q}\right)^{i} I_{a}\right] \quad[\mathcal{O}(0.1) S-P] \\
& (--++) \frac{F_{P S}\left(q^{2}\right) F_{A}\left(q^{2}\right)}{4 m_{p}}\left[\sigma_{a}^{i}\left(\boldsymbol{\sigma}_{b} \cdot \mathbf{q}\right)+\sigma_{b}^{i}\left(\boldsymbol{\sigma}_{a} \cdot \mathbf{q}\right)\right] \quad[\mathcal{O}(10) S-P] \\
& (-++-) \frac{F_{P S}\left(q^{2}\right) F_{V}\left(q^{2}\right)}{8 m_{p}^{2}} Q^{i}\left[I_{a}\left(\boldsymbol{\sigma}_{b} \cdot \mathbf{q}\right)+I_{b}\left(\boldsymbol{\sigma}_{a} \cdot \mathbf{q}\right)\right] \quad[\mathcal{O}(1) S-P] \\
& (+-+-) \frac{F_{S}\left(q^{2}\right) F_{P}\left(q^{2}\right)}{8 m_{p}^{2}} q^{i}\left[\left(\boldsymbol{\sigma}_{a} \cdot \mathbf{q}\right) I_{b}+\left(\boldsymbol{\sigma}_{b} \cdot \mathbf{q}\right) I_{a}\right] \quad[\mathcal{O}(1) S-P] \\
& \left.(++--) \frac{F_{P S}\left(q^{2}\right) F_{P}\left(q^{2}\right)}{8 m_{p}^{3}} q^{i}\left(\boldsymbol{\sigma}_{a} \cdot \mathbf{q}\right)\left(\boldsymbol{\sigma}_{b} \cdot \mathbf{q}\right)\right\}+\cdots . \quad[\mathcal{O}(10) S-P]
\end{aligned}
$$

\section{APPENDIX B: LEPTONIC MATRIX ELEMENTS}

Terms 1, 2, and 3.-The electron current for these terms is

$$
j=\bar{e}_{1}(x)\left(1 \pm \gamma_{5}\right) e_{2}^{c}(x)
$$

Note that both the electron wave functions depend on the same coordinate variable, as a contact interaction is considered. In the $S_{1 / 2}-S_{1 / 2}$ wave approximation and using Tomoda's notation, we obtain

$$
\begin{aligned}
\bar{e}_{1}\left(1 \pm \gamma_{5}\right) e_{2}^{c} & \approx\left(\bar{e}_{\mathbf{p}_{1} s}\right)^{S_{1 / 2}}\left(1 \pm \gamma_{5}\right)\left(e_{\mathbf{p}_{2} s^{\prime}}^{c}\right)^{S_{1 / 2}} \\
& =\left(e_{\mathbf{p}_{1} s}^{S_{1 / 2}}\right)^{\dagger} \gamma_{0}\left(1 \pm \gamma_{5}\right) i \gamma_{2}\left(e_{\mathbf{p}_{2} s^{\prime}}^{S_{1 / 2}}{ }^{*}\right. \\
& =\left(g_{-1}\left(\epsilon_{1}, r\right) \chi_{s}^{\dagger} \quad f_{1}\left(\epsilon_{1}, r\right) \chi_{s}^{\dagger}\left(\boldsymbol{\sigma} \cdot \hat{\mathbf{p}}_{1}\right)\right) \gamma_{0}\left(1 \pm \gamma_{5}\right) i \gamma_{2}\left(\begin{array}{c}
g_{-1}\left(\epsilon_{2}, r\right) \chi_{s^{\prime}} \\
f_{1}\left(\epsilon_{2}, r\right)\left(\boldsymbol{\sigma} \cdot \hat{\mathbf{p}}_{2}\right) \chi_{s^{\prime}}
\end{array}\right),
\end{aligned}
$$

where all the matrices are considered in the standard Dirac representation,

$$
\gamma_{0}=\left(\begin{array}{cc}
1 & 0 \\
0 & -1
\end{array}\right), \quad \gamma=\left(\begin{array}{cc}
0 & \sigma \\
-\sigma & 0
\end{array}\right), \quad \gamma_{5}=\left(\begin{array}{cc}
0 & 1 \\
1 & 0
\end{array}\right), \quad C=i \gamma_{2} \gamma_{0}=\left(\begin{array}{cc}
0 & -i \sigma_{2} \\
-i \sigma_{2} & 0
\end{array}\right)
$$

Next, we expand and square Eq. (B2). After summing over spins and using the properties of the spinors $\chi_{s}$, we get 


$$
\begin{aligned}
& \sum_{s, s^{\prime}}\left[\left(f^{-1} \chi_{1}^{\dagger}\left(\boldsymbol{\sigma} \cdot \hat{\mathbf{p}}_{2}\right) \sigma_{2} \chi_{s^{\prime}}+f_{1}^{-1} \chi_{s}^{\dagger}\left(\boldsymbol{\sigma} \cdot \hat{\mathbf{p}}_{1}\right) \sigma_{2} \chi_{s^{\prime}}\right)\right. \\
& \left.\quad \pm\left(f^{-1-1} \chi_{s}^{\dagger} \sigma_{2} \chi_{s^{\prime}}+f_{11} \chi_{s}^{\dagger}\left(\boldsymbol{\sigma} \cdot \hat{\mathbf{p}}_{1}\right)\left(\boldsymbol{\sigma} \cdot \hat{\mathbf{p}}_{2}\right) \sigma_{2} \chi_{s^{\prime}}\right)\right]^{2} \\
& =2\left[f_{11}^{(0)}+f_{11+}^{(1)}\left(\hat{\mathbf{p}}_{1} \cdot \hat{\mathbf{p}}_{2}\right)\right],
\end{aligned}
$$

where $f_{11}^{(0)}$ and $f_{11+}^{(1)}$ are defined in Eq. (89). For the interference term combining a left-handed with a right-handed electron current, the calculation is analogous to the procedure shown above, but the plus sign in the definition of $f_{11}^{(1)}$ would change to a minus sign.

Terms 4 and 5.-The electron current for these terms is

$$
j^{\mu} \equiv \overline{e_{1}}(x) \gamma^{\mu} \gamma_{5} e_{2}^{c}(x) .
$$

In the $S_{1 / 2}-S_{1 / 2}$ approximation, we have

$$
\begin{aligned}
& \overline{e_{1}} \gamma^{\mu} \gamma_{5} e_{2}^{c} \approx\left(\bar{e}_{\mathbf{p}_{1} s}\right)^{S_{1 / 2}} \gamma_{\mu} \gamma_{5}\left(e_{\mathbf{p}_{2} s^{\prime}}^{c}\right)^{S_{1 / 2}} \\
& =\left(e_{\mathbf{p}_{1} s}^{S_{1 / 2}}\right)^{\dagger} \gamma_{0} \gamma_{\mu} \gamma_{5} i \gamma_{2}\left(e_{\mathbf{p}_{2} s^{\prime}}^{S_{1 / 2}}\right)^{*} \\
& =\left(\begin{array}{ll}
g_{-1}\left(\epsilon_{1}, r\right) \chi_{s}^{\dagger} & f_{1}\left(\epsilon_{1}, r\right) \chi_{s}^{\dagger}\left(\boldsymbol{\sigma} \cdot \hat{\mathbf{p}}_{1}\right)
\end{array}\right) \gamma_{0} \gamma_{\mu} \gamma_{5} i \gamma_{2}\left(\begin{array}{c}
g_{-1}\left(\epsilon_{2}, r\right) \chi_{s^{\prime}} \\
f_{1}\left(\epsilon_{2}, r\right)\left(\boldsymbol{\sigma} \cdot \hat{\mathbf{p}}_{2}\right) \chi_{s^{\prime}}
\end{array}\right) .
\end{aligned}
$$

For $\mu=0$, after squaring, summing over spins, and using the properties of the spinors $\chi_{s}$, we obtain

$$
\sum_{s, s^{\prime}}\left[f^{-1-1} \chi_{s}^{\dagger} \sigma_{2} \chi_{s^{\prime}}+f_{11} \chi_{s}^{\dagger}\left(\boldsymbol{\sigma} \cdot \hat{\mathbf{p}}_{1}\right)\left(\boldsymbol{\sigma} \cdot \hat{\mathbf{p}}_{2}\right) \sigma_{2} \chi_{s^{\prime}}\right]^{2}=\frac{1}{8}\left[f_{66}^{(0)}+f_{66}^{(1)}\left(\hat{\mathbf{p}}_{1} \cdot \hat{\mathbf{p}}_{2}\right)\right]
$$

where $f_{66}^{(0)}$ and $f_{66}^{(1)}$ are defined in Eq. (90). A similar derivation is possible for spatial $\mu=k$; however, as stated in the main text, it does not enter the contributions to $0^{+} \rightarrow 0^{+}$transition.

Interference between terms 1,2,3 and 4,5.-For the interference between terms 1,2,3 and terms 4,5, we use the same procedure as before, and for $\mu=0$ we obtain

$$
\begin{aligned}
& \sum_{s, s^{\prime}}\left[f^{-1-1} \chi_{s}^{\dagger} \sigma_{2} \chi_{s^{\prime}}+f_{11} \chi_{s}^{\dagger}\left(\boldsymbol{\sigma} \cdot \hat{\mathbf{p}}_{1}\right)\left(\boldsymbol{\sigma} \cdot \hat{\mathbf{p}}_{2}\right) \sigma_{2} \chi_{s^{\prime}}\right]^{\dagger}\left[\left(f^{-1} \chi_{s}^{\dagger}\left(\boldsymbol{\sigma} \cdot \hat{\mathbf{p}}_{2}\right) \sigma_{2} \chi_{s^{\prime}}+f_{1}^{-1} \chi_{s}^{\dagger}\left(\boldsymbol{\sigma} \cdot \hat{\mathbf{p}}_{1}\right) \sigma_{2} \chi_{s^{\prime}}\right)\right. \\
& \left.\quad \pm\left(f^{-1-1} \chi_{s}^{\dagger} \sigma_{2} \chi_{s^{\prime}}+f_{11} \chi_{s}^{\dagger}\left(\boldsymbol{\sigma} \cdot \hat{\mathbf{p}}_{1}\right)\left(\boldsymbol{\sigma} \cdot \hat{\mathbf{p}}_{2}\right) \sigma_{2} \chi_{s^{\prime}}\right)\right] \\
& =\mp \frac{1}{2}\left[f_{16}^{(0)}+f_{16}^{(1)}\left(\hat{\mathbf{p}}_{1} \cdot \hat{\mathbf{p}}_{2}\right)\right],
\end{aligned}
$$

where $f_{16}^{(0)}$ and $f_{16}^{(1)}$ are defined in Eq. (91). As before, we do not present the phase-space factor for spatial $\mu=k$, as it does not enter the calculation of $0^{+} \rightarrow 0^{+}$transitions.

[1] G. Aad et al. (ATLAS Collaboration), Phys. Lett. B 716, 1 (2012).

[2] S. Chatrchyan et al. (CMS Collaboration), Phys. Lett. B 716, 30 (2012).

[3] K. A. Olive et al. (Particle Data Group), Chin. Phys. C 38, 090001 (2014).

[4] P. A. R. Ade et al. (Planck Collaboration), Astron. Astrophys. 594, A13 (2016).

[5] S. Weinberg, Phys. Rev. Lett. 43, 1566 (1979).

[6] P. Minkowski, Phys. Lett. 67B, 421 (1977).

[7] R. N. Mohapatra and G. Senjanovic, Phys. Rev. Lett. 44, 912 (1980).
[8] T. Yanagida, Conf. Proc. C 7902131, 95 (1979).

[9] M. Gell-Mann, P. Ramond, and R. Slansky, Conf. Proc. C 790927, 315 (1979).

[10] J. Schechter and J. W. F. Valle, Phys. Rev. D 22, 2227 (1980).

[11] J. Schechter and J. Valle, Phys. Rev. D 25, 2951 (1982).

[12] H. Päs, M. Hirsch, H. Klapdor-Kleingrothaus, and S. Kovalenko, Phys. Lett. B 453, 194 (1999).

[13] H. Pas, M. Hirsch, H. V. Klapdor-Kleingrothaus, and S. G. Kovalenko, Phys. Lett. B 498, 35 (2001).

[14] F. del Aguila, A. Aparici, S. Bhattacharya, A. Santamaria, and J. Wudka, J. High Energy Phys. 05 (2012) 133. 
[15] F. del Aguila, A. Aparici, S. Bhattacharya, A. Santamaria, and J. Wudka, J. High Energy Phys. 06 (2012) 146.

[16] F. Simkovic, G. Pantis, J. D. Vergados, and A. Faessler, Phys. Rev. C 60, 055502 (1999).

[17] F. F. Deppisch, L. Graf, J. Harz, and W.-C. Huang, Phys. Rev. D 98, 055029 (2018).

[18] F. F. Deppisch, J. Harz, M. Hirsch, W.-C. Huang, and H. Päs, Phys. Rev. D 92, 036005 (2015).

[19] F. F. Deppisch, J. Harz, and M. Hirsch, Phys. Rev. Lett. 112, 221601 (2014).

[20] F. F. Deppisch and J. Harz, arXiv:1408.5351.

[21] A. Gando et al. (KamLAND-Zen Collaboration), Phys. Rev. Lett. 117, 082503 (2016); 117, 109903(E) (2016).

[22] B. Pontecorvo, Phys. Lett. 26B, 630 (1968).

[23] C. Giunti and M. Laveder, Phys. Rev. D 82, 053005 (2010).

[24] C. Giunti, M. Laveder, Y. F. Li, and H. W. Long, Phys. Rev. D 88, 073008 (2013).

[25] J. Barry, W. Rodejohann, and H. Zhang, J. High Energy Phys. 07 (2011) 091.

[26] J. Barea, J. Kotila, and F. Iachello, Phys. Rev. D 92, 093001 (2015).

[27] M. Blennow, E. Fernandez-Martinez, J. Lopez-Pavon, and J. Menendez, J. High Energy Phys. 07 (2010) 096.

[28] A. Faessler, M. Gonzalez, S. Kovalenko, and F. Simkovic, Phys. Rev. D 90, 096010 (2014).

[29] F. Deppisch, H. Päs, and J. Suhonen, Phys. Rev. D 72, 033012 (2005).

[30] J. Barea and F. Iachello, Phys. Rev. C 79, 044301 (2009).

[31] J. Barea, J. Kotila, and F. Iachello, Phys. Rev. C 87, 014315 (2013).

[32] J. Barea, J. Kotila, and F. Iachello, Phys. Rev. C 91, 034304 (2015).

[33] F. Simkovic, A. Faessler, V. Rodin, P. Vogel, and J. Engel, Phys. Rev. C 77, 045503 (2008).

[34] F. Simkovic, V. Rodin, A. Faessler, and P. Vogel, Phys. Rev. C 87, 045501 (2013).

[35] J. Suhonen, J. Phys. G 19, 139 (1993).

[36] J. Suhonen, AIP Conf. Proc. 1488, 326 (2012).

[37] E. Caurier, F. Nowacki, and A. Poves, Int. J. Mod. Phys. E 16, 552 (2007).

[38] J. Menendez, A. Poves, E. Caurier, and F. Nowacki, Nucl. Phys. A818, 139 (2009).

[39] T. R. Rodriguez and G. Martinez-Pinedo, Phys. Rev. Lett. 105, 252503 (2010).

[40] M. Agostini et al., Nature (London) 544, 47 (2017).

[41] M. Agostini, G. Benato, and J. Detwiler, Phys. Rev. D 96, 053001 (2017).

[42] B. Mong et al. (nEXO Collaboration), Proc. Sci. HQL2016 (2017) 074.

[43] F. F. Deppisch, M. Hirsch, and H. Päs, J. Phys. G 39, 124007 (2012).

[44] J. D. Vergados, H. Ejiri, and F. Simkovic, Int. J. Mod. Phys. E 25, 1630007 (2016).

[45] J. Engel and J. Menendez, Rep. Prog. Phys. 80, 046301 (2017).

[46] M. Doi, T. Kotani, H. Nishiura, K. Okuda, and E. Takasugi, Phys. Theor. Phys. 66, 1739 (1981).

[47] M. Doi, T. Kotani, H. Nishiura, and E. Takasugi, Phys. Theor. Phys. 69, 602 (1983).
[48] T. Tomoda, Rep. Prog. Phys. 54, 53 (1991).

[49] A. Ali, A. Borisov, and D. Zhuridov, arXiv:hep-ph/ 0606072.

[50] A. Ali, A. V. Borisov, and D. V. Zhuridov, Phys. Rev. D 76, 093009 (2007).

[51] J. C. Pati and A. Salam, Phys. Rev. D 10, 275 (1974).

[52] R. N. Mohapatra and J. C. Pati, Phys. Rev. D 11, 566 (1975).

[53] R. N. Mohapatra and J.C. Pati, Phys. Rev. D 11, 2558 (1975).

[54] G. Senjanovic and R. N. Mohapatra, Phys. Rev. D 12, 1502 (1975).

[55] J. C. Helo, M. Hirsch, and T. Ota, J. High Energy Phys. 06 (2016) 006.

[56] L. Lehman, Phys. Rev. D 90, 125023 (2014).

[57] B. Henning, X. Lu, T. Melia, and H. Murayama, J. High Energy Phys. 08 (2017) 016.

[58] M. L. Graesser, J. High Energy Phys. 08 (2017) 099.

[59] G. Prezeau, M. Ramsey-Musolf, and P. Vogel, Phys. Rev. D 68, 034016 (2003).

[60] T. Tomoda and A. Faessler, Phys. Lett. B 199, 475 (1987).

[61] J. Kotila and F. Iachello, Phys. Rev. C 85, 034316 (2012).

[62] S. L. Adler, E. W. Colglazier, J. B. Healy, I. Karliner, J. Lieberman, Y. J. Ng, and H.-S. Tsao, Phys. Rev. D 11, 3309 (1975).

[63] S. Weinberg, Phys. Rev. 112, 1375 (1958).

[64] R. Bijker and F. Iachello, Phys. Rev. C 69, 068201 (2004).

[65] W. M. Yao et al. (Particle Data Group), J. Phys. G 33, 1 (2006).

[66] M. R. Schindler and S. Scherer, Eur. Phys. J. A 32, 429 (2007);

[67] V. Bernard, L. Elouadrhiri, and U.-G. Meissner, J. Phys. G 28, R1 (2002).

[68] V. A. Andreev et al. (MuCap Collaboration), Phys. Rev. Lett. 110, 012504 (2013).

[69] M. González-Alonso, O. Naviliat-Cuncic, and N. Severijns, arXiv:1803.08732.

[70] L. L. Foldy and S. A. Wouthuysen, Phys. Rev. 78, 29 (1950).

[71] M. E. Rose and R. K. Osborn, Phys. Rev. 93, 1315 (1954).

[72] E. Caurier, J. Menendez, F. Nowacki, and A. Poves, Phys. Rev. Lett. 100, 052503 (2008).

[73] G. A. Miller and J. E. Spencer, Ann. Phys. (N.Y.) 100, 562 (1976).

[74] F. Simkovic, A. Faessler, H. Muther, V. Rodin, and M. Stauf, Phys. Rev. C 79, 055501 (2009).

[75] M. Agostini et al. (GERDA Collaboration), Phys. Rev. Lett. 111, 122503 (2013).

[76] M. Martinez et al. (CUORE Collaboration), Status of CUORE: An observatory for neutrinoless double beta decay and other rare events, in Proceedings of 12th Patras Workshop on Axions, WIMPs and WISPs (PATRAS 2016): Jeju Island, South Korea, 2016 (Verlag Deutsches Elektronen-Synchrotron, Hamburg, 2017), pp. 112-115.

[77] A. Gando et al. (KamLAND-Zen Collaboration), Phys. Rev. Lett. 110, 062502 (2013).

[78] M. González, M. Hirsch, and S. G. Kovalenko, Phys. Rev. D 93, 013017 (2016); 97, 099907(E) (2018).

[79] V. Cirigliano, W. Dekens, J. de Vries, M. L. Graesser, and E. Mereghetti, arXiv:1806.02780. 